\title{
COMPOSITE DYNAMIC MODELS FOR PERIODICALLY HETEROGENEOUS MEDIA
}

\author{
Daniel J. Colquitt ${ }^{1}$, Vladyslav V. Danishevskyy²,*, Julius D. Kaplunov² \\ ${ }^{1}$ Department of Mathematical Sciences, University of Liverpool, UK \\ ${ }^{2}$ School of Computing and Mathematics, Keele University, UK
}

\begin{abstract}
Propagation of elastic waves through discrete and continuous periodically heterogeneous media is studied. A two-scale asymptotic procedure allows us to derive macroscopic dynamic equations applicable at frequencies close to the resonant frequencies of the unit cells. Matching the asymptotic solutions by two-point Padé approximants, we obtain new higher-order equations that describe the dynamic behaviour of the medium both in the low and in the high frequency limits. An advantage of the proposed approach is that all the macroscopic parameters can be determined explicitly in terms of the microscopic properties of the medium. Dispersion diagrams are evaluated and the propagation of transient waves induced by pulse and harmonic loads is considered. The developed analytical models are verified by comparison with data of numerical simulations. For high-contrast media, we can observe an analogy between the propagation of waves in heterogeneous solids and in thin walled waveguides. It is also shown that different combinations of cell resonances may uprise some additional types of waves that do not appear in the classical continuous theory.
\end{abstract}

Key words: wave propagation; heterogeneous media; phononic bands; dispersion; asymptotic homogenisation; two-scale perturbations; Padé approximants; transient waves.

\section{Introduction}

In the last years there has been a rapid upsurge in scholarly interest in the dynamic response of multi-scale heterogeneous media and structures. This increased attention is motivative, primarily, by numerous applications to mechanical composites and metamaterials (see, for example, a reviewer by Zheludev and Kivshar, 2012). Intensive studies were devoted to phononic band gaps

* Corresponding author. E-mail address: v.danishevskyy@keele.ac.uk (for V.V. Danishevskyy). 
(Sigalas and Economou, 1993; Kushwaha et al., 1994; Nicorovici et al., 1995), negative refraction and flat lenses (Pendry, 2000; Grbic and Eleftheriades, 2004; Colquitt et al., 2011), dynamic anisotropy and waves focusing (Ayzenberg-Stepanenko and Slepyan, 2008; Colquitt et al., 2012), acoustic diodes (Liang et al., 2009), acoustically invisible cloaks (Milton et al., 2006; Norris and Shuvalov, 2011; Colquitt et al., 2013), waves localisation in structures with defects (Craster et al., 2010b; Colquitt et al., 2011; Andrianov et al., 2013, 2014), topologically protected edge waves (Wang, 2015), chirality (Carta, 2017). Recent progress in the field is outlined in review papers by Maldovan (2013) and Hussein et al. (2014).

The dynamic behaviour of heterogeneous media can be described by gradient continuum theories. According to them, the influence of the microstructure is modelled phenomenologically by allowing the medium some additional internal degrees of freedom. The origins of the gradient elasticity are traced back to the very early developments by Cauchy (1851) and Voigt (1887), who studied discrete lattice models. Gradient theories for elastic continua were introduced by Cosserat (1909) and Le Roux (1911). Later on, various models were developed and specified by Aero and Kuvshinskii (1961), Toupin (1962), Mindlin and Tiersten (1962), Kunin (1966), Herrmann and Achenbach (1968), Levin (1971), and many others. Gradient models can be also derived from integral non-local theories (Eringen, 1983). The reader is referred to Askes and Aifantis (2011) and to Madeo (2015) for an overview of generalised theories of elasticity.

A disadvantage of generalised continuum theories is that they are phenomenological in the sense that the relationship between micro- and macroscale properties of the system is usually not known a priori. The macroscopic parameters are expected to be deduced from experimental observations and this inverse problem, in general, may be very difficult if not impossible.

An alternative approach to model the dynamic response of heterogeneous media is provided by the two-scale asymptotic homogenisation method. It involves a scaling of spatial variables by a small parameter $\varepsilon$ which characterises the size of the microstructure. Then scaled and unscaled variables are treated as independent from each other and unknown physical fields (e.g., displacements) are introduced as asymptotic expansions in ascending powers of $\varepsilon$. This generates a recurrent hierarchy of cell problems, representing perturbations away from the leading-order approximation. Cell problems describe local variations of the physical fields on the micro scale. For one-dimensional structures they are usually solved analytically, while for two- and threedimensional models numerical procedures can be applied. Then integration over the unit cell domain provides us with homogenised (so called effective) macroscopic equations that describe the behaviour of the system on scales much larger than the characteristic length of the microstructure.

The origins of the asymptotic homogenisation has been documented by Bensoussan et al. 
(1978), Sánchez-Palencia (1980), Bakhvalov and Panasenko (1989) primarily focusing on the quasi-static limit. Extensions to dynamic problems were proposed by Boutin and Auriault (1993), Fish and Chen (2001), Andrianov et al. (2008), Soubestre and Boutin (2012) Auriault and Boutin (2012). Typically, asymptotic homogenisation is applied to instances where there is a large discrepancy between the characteristic length-scale of the microstructure and the wavelength of waves propagating through the material.

More recently, the method of high-frequency homogenisation was introduced as a powerful tool for the analysis of the dynamic response of metamaterial structures. Developed initially for second-order scalar equations (Daya and Potier-Ferry, 2001; Craster, 2010a; Craster, 2010b; Craster et al., 2012) and later extended to more sophisticated setups (Antonakakis, 2014; Colquitt, 2015), this approach is similar to the classical asymptotic homogenisation. But rather than perturbing away from the static limit, it involves perturbations away from the cell resonances. As a result, the obtained macroscopic equations appear to be applicable for the wavelength comparable to the length-scale of the microstructure.

An important advantage of the two-scale asymptotic homogenisation is that relationships between the microscopic and macroscopic parameters can be predicted on a rigorous theoretical basis. The obtained macroscopic models encapsulate information about the microstructure and all their coefficients can be determined from the geometry of the unit cell and the properties of the constitutive components.

Here we develop the techniques of asymptotic homogenisation to derive effective partial differential equations that describe the macroscopic dynamic behaviour of heterogeneous media and structures. The two-scale asymptotic procedure provides us with the solutions applicable in the vicinity of the resonant frequencies of the unite cells. Then, using the method of two-point Padé approximants (Baker and Graves-Morris, 1985), we match limiting asymptotic solutions and derive new macroscopic models shown to be valid in a wide range of frequencies. The effective dynamic parameters are given explicitly in terms of the microscopic properties of the medium. This contrasts with the classical generalised continuum theories, where the material parameters are usually left undetermined. The methods established and illustrated in the present paper offer a more direct approach to the development of generalised theories of continua. In particular, the macroscopic models follow as a consequence of and are derived directly from the microstructural properties of the medium rather than, as in the classical approach to generalised continua, the macroscopic model is postulated and then an inverse problem has to be solved to deduce the corresponding microscopic properties.

The paper is organised as follows. In order to distil the essential elements of the 
methodology, we begin in Section 2 by considering wave propagation in a discrete one-dimensional lattice. The dispersive properties and the exact solutions are studied in detail. We then proceed to develop the two-scale asymptotic approach and to apply Padé approximants in order to derive the effective macroscopic equation valid in both the low and the high frequency limits. The obtained equation is verified simulating a transient wave propagation in in the lattice subjected to pulse and harmonic loads. The developed analytical model exhibits excellent agreement with the direct numerical solution.

In Section 3, we extend the proposed approach to continuous media, although, for the sake of clarity we remain in the one-dimensional framework. In particular, a piecewise homogeneous string is considered and its shown that the approach developed in Section 2 can be applied mutatis mutandis. We also study an important case of high-contrast media and highlight a qualitative analogy between the dynamic behaviour of heterogeneous solids and thin walled structures (Craster et al., 2014; Kaplunov, 2017).

In Section 4, a generalisation of the developed approaches to two-dimensional structures is discussed. We consider anti-plane waves in a square lattice and demonstrate that the increased dimension of the problem allows for generating a range of various higher-order macroscopic equations based on different combinations of cell resonances.

Finally, we draw together a few concluding remarks in Section 5.

\section{Monatomic lattice}

\subsection{Discrete model}

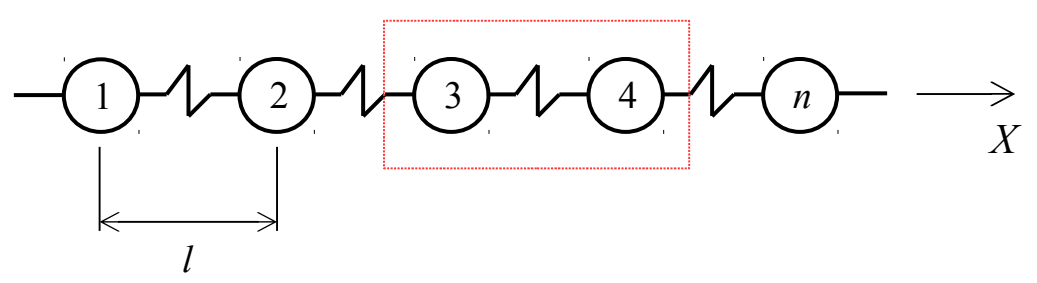

Fig. 1. Monatomic lattice with a two-particles unit cell.

We start with the classical problem of wave propagation through an infinite lattice consisting of identical particles of the mass $m$ connected by massless springs of rigidity $c$ (Fig. 1). This well known model can be used to describe vibrations in solid-state crystals (Kittel, 1996) and cellular structures (Gibson et al., 1997); it also finds application in some engineering problems, such as the analysis of the dynamics of lightweight strings with attached masses (like overhead 
power cables) or interactions of railway coaches.

Let us introduce a unit cell that includes two particles. We use different notations $u_{n}^{(1)}$ and $u_{n}^{(2)}$ for the displacements of odd and even particles accordingly, i.e.

$$
u_{n}=\left\{\begin{array}{l}
u_{n}^{(1)} \text { for } n= \pm 1, \pm 3, \pm 5, \ldots ; \\
u_{n}^{(2)} \text { for } n=0, \pm 2, \pm 4, \ldots ;
\end{array}\right.
$$

where $n$ is the number of the particle. Vasiliev et al. (2010) referred this approach as a two-field model. The equations of motion for the particles in the unit cell take the form:

$$
\begin{aligned}
& m \frac{d^{2} u_{n}^{(1)}}{d T^{2}}+c\left(2 u_{n}^{(1)}-u_{n+1}^{(2)}-u_{n-1}^{(2)}\right)=0 \quad \text { for } \quad n= \pm 1, \pm 3, \pm 5, \ldots ; \\
& m \frac{d^{2} u_{n}^{(2)}}{d T^{2}}+c\left(2 u_{n}^{(2)}-u_{n+1}^{(1)}-u_{n-1}^{(1)}\right)=0 \quad \text { for } \quad n=0, \pm 2, \pm 4, \ldots ;
\end{aligned}
$$

where $T$ is time.

Considering a time-harmonic wave, we suppose that particles in the unit cell can move with different amplitudes $A_{1}$ and $A_{2}$, i.e.

$$
\begin{aligned}
& u_{n}^{(1)}=A_{1} \exp (-i k n) \exp (i \Omega T), \\
& u_{n}^{(2)}=A_{2} \exp (-i k n) \exp (i \Omega T),
\end{aligned}
$$

where $\Omega$ is the angular frequency and $k$ is the non-dimensional wave number. Substituting (2) into (1), one comes to a system of equations for the unknown amplitudes $A_{1}, A_{2}$ :

$$
\begin{aligned}
& \left(2-\omega^{2}\right) A_{1}-2 \cos (k) A_{2}=0, \\
& -2 \cos (k) A_{1}+\left(2-\omega^{2}\right) A_{2}=0,
\end{aligned}
$$

where $\omega$ is the non-dimensional angular frequency, $\omega=\Omega(m / c)^{1 / 2}$.

System (3) has a non-trivial solution if and only if the determinant of the matrix of the coefficients is zero. This condition results in the dispersion equation: 


$$
\left(2-\omega^{2}\right)^{2}-4 \cos ^{2}(k)=0 .
$$

System (3) also provides us with the relation between the amplitudes of the particles:

$$
\frac{A_{1}}{A_{2}}=\frac{2-\omega^{2}}{2 \cos (k)} .
$$

Equation (4) has two different roots for the dispersion curves. The first root is

$$
\omega^{2}=4 \sin ^{2}(k / 2)
$$

It describes propagation of the conventional acoustic mode. In this case expression (5) gives $A_{1}=A_{2}$ and the particles in the unit cell move in-phase. In the long-wave limit, as $k \rightarrow 0$, one obtains $u_{n}^{(1)} \approx u_{n+1}^{(2)}$, so the wave is periodic across the unit cell. We refer this type of wave as a periodic mode.

The second root of the dispersion equation (4) is

$$
\omega^{2}=4 \cos ^{2}(k / 2)
$$

which implies $A_{1}=-A_{2}$. Opposite to the previous case, the neighbouring particles move out-ofphase and in the long-wave limit the solution is anti-periodic across the unit cell: $u_{n}^{(1)} \approx-u_{n+1}^{(2)}$ as $k \rightarrow 0$. We shall call this wave an anti-periodic mode.

The dispersion curves are shown at Fig. 2. In the frequency range $0 \leq \omega \leq 2$, there are two acoustic branches for the periodic and anti-periodic wave accordingly. At $\omega>2$ the wave number $k$ becomes complex and the spectrum of the lattice exhibits a band gap and the signal decays exponentially with an attenuation coefficient equal to the imaginary part of the wave number. It should be noted that the periodic mode exhibits normal dispersion having positive group velocity, while the anti-periodic mode displays abnormal dispersion and propagates with negative group velocity.

Displacements of the particles for periodic and anti-periodic modes are shown in Fig. 3. In the long-wave limit the frequency of the periodic mode vanishes, $\omega \rightarrow 0$ as $k \rightarrow 0$, so no vibrations occur and the motion is simply a rigid body translation. In contrast, the anti-periodic mode becomes a standing wave with zero group velocity and non-zero frequency: $\omega \rightarrow 2$ as $k \rightarrow 0$. This regime 
can be considered as a "hidden" or "trapped" mode, in the sense that no energy is transmitted on macro scale, but on micro scale the lattice exhibits saw-tooth oscillations. Such trapped modes do not exist in the framework of classical continuous theory.

Finally, we also note that the dispersion relations (6) and (7) obtained for the periodic and anti-periodic modes are a reflection of each other in the line $k=\pi / 2$.

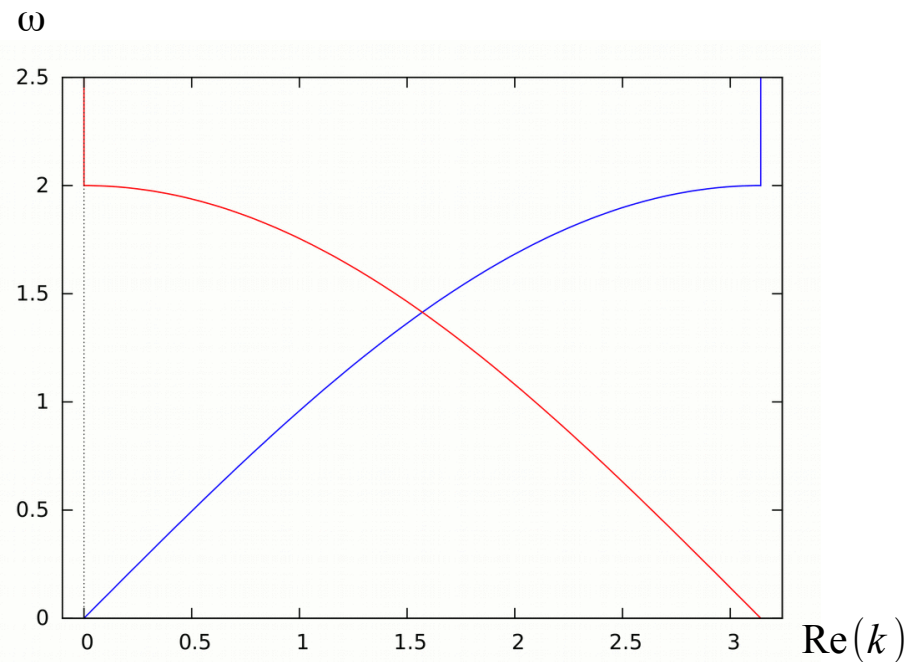

Fig. 2. Dispersion curves of the monatomic lattice.

Blue - periodic mode; red - anti-periodic mode.

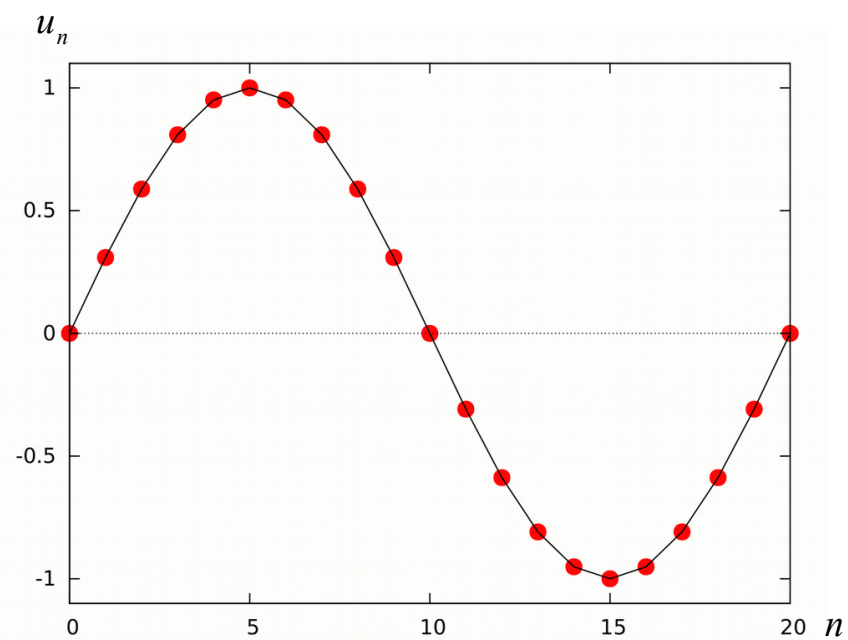

a) periodic mode 


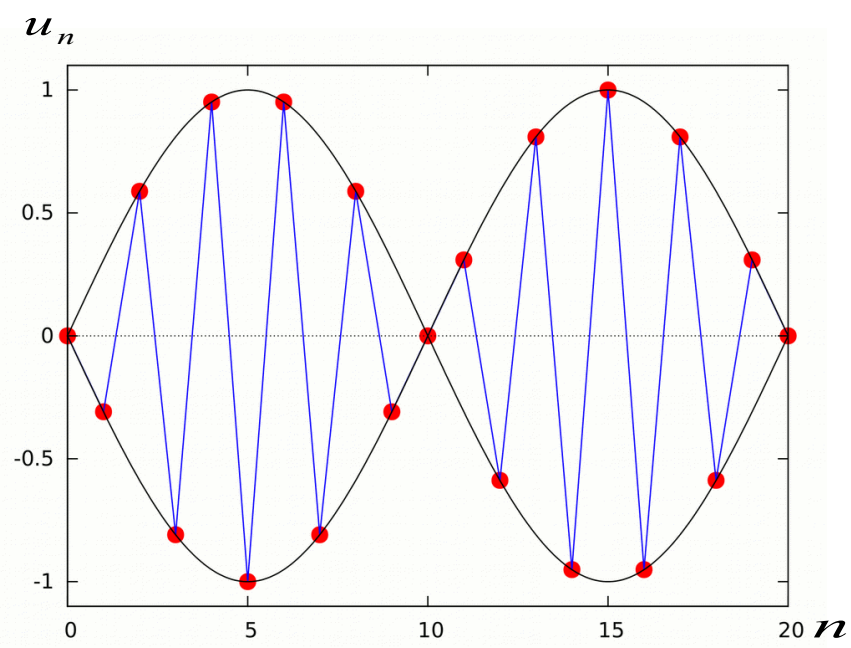

b) anti-periodic mode

Fig. 3. Displacements of particles; $k=\pi / 10$. Here the real parts of the complex form expressions (2) are considered.

\subsection{Asymptotic continuous approximations}

In the previous sub-section, explicit analytical expressions for the dispersion curves and mode shapes have been presented. Now we turn to the problem of a continuous approximation of the discrete model. Our aim is to obtain macroscopic differential equations that describe, asymptotically, the propagation of periodic and anti-periodic modes. We note that the periodic case is classical and have been treated many times before (see, for example, Andrianov et al. (2010) and references therein).

In the framework of the two-field model presented above, let us introduce the effective wave field $\tilde{u}_{n}$ describing propagation of the envelope wave (the black curves in Fig. 3). For the periodic mode we denote

$$
\begin{aligned}
& \widetilde{u}_{n}=\frac{u_{n}^{(1)}+u_{n+1}^{(2)}}{2} \text { for } n= \pm 1, \pm 3, \pm 5, \ldots, \\
& \widetilde{u}_{n}=\frac{u_{n+1}^{(1)}+u_{n}^{(2)}}{2} \text { for } n=0, \pm 2, \pm 4, \ldots ;
\end{aligned}
$$

while for the anti-periodic mode one has 


$$
\begin{gathered}
\widetilde{u}_{n}=\frac{u_{n}^{(1)}-u_{n+1}^{(2)}}{2} \text { for } n= \pm 1, \pm 3, \pm 5, \ldots, \\
\widetilde{u}_{n}=\frac{u_{n+1}^{(1)}-u_{n}^{(2)}}{2} \text { for } n=0, \pm 2, \pm 4, \ldots
\end{gathered}
$$

With respect to the new field function $\widetilde{u}_{n}$, equations (1) become uncoupled and read

$$
m \frac{d^{2} \widetilde{u}_{n}}{d T^{2}}+c\left(2 \widetilde{u}_{n}-\widetilde{u}_{n+1}-\widetilde{u}_{n-1}\right)=0
$$

for the periodic mode and

$$
m \frac{d^{2} \widetilde{u}_{n}}{d T^{2}}+c\left(2 \widetilde{u}_{n}+\widetilde{u}_{n+1}+\widetilde{u}_{n-1}\right)=0
$$

for the anti-periodic case.

Let us introduce a spatially continuous function $u(X, T)$ describing the displacement of the lattice:

$$
\left.u(X, T)\right|_{X=n l}=\tilde{u}_{n}(T),
$$

where $l$ is the distance between the particles. We seek for a long-wave asymptotic approximation, supposing the wavelength $L$ to be much larger then the size $l$ of the microstructure. Then a natural small parameter can be introduced as follows: $\varepsilon=l / L$, where $0<\varepsilon \ll 1$. We also define a non-dimensional spatial co-ordinate $x=X / L$. Using a Taylor series expansion, we may write

$$
\widetilde{u}_{n \pm 1}(T)=u(x \pm \varepsilon, T)=\sum_{p=0}^{\infty} \frac{( \pm \varepsilon)^{p}}{p !} \frac{\partial^{p} u(x, T)}{\partial x^{p}}
$$

Substituting (10) and (11) into (8) and (9), we obtain continuous macroscopic equations that describe the dynamics of the lattice in the long-wave limit as $\varepsilon \rightarrow 0$. Within the error $O\left(\varepsilon^{6}\right)$ they read 
for periodic mode

$$
\varepsilon^{2} \frac{\partial^{2} u}{\partial x^{2}}+\frac{1}{12} \varepsilon^{4} \frac{\partial^{4} u}{\partial x^{4}}-\frac{\partial^{2} u}{\partial t^{2}}=0
$$

for anti-periodic mode

$$
4 u+\varepsilon^{2} \frac{\partial^{2} u}{\partial x^{2}}+\frac{1}{12} \varepsilon^{4} \frac{\partial^{4} u}{\partial x^{4}}+\frac{\partial^{2} u}{\partial t^{2}}=0
$$

where $t$ is the non-dimensional time, $t=T(\mathrm{c} / \mathrm{m})^{1 / 2}$.

For the further analysis, it is convenient to write equations (12) and (13) with respect to a non-dimensional spatial variable $y=X / l$ :

$$
\begin{gathered}
\frac{\partial^{2} u}{\partial y^{2}}+\frac{1}{12} \frac{\partial^{4} u}{\partial y^{4}}-\frac{\partial^{2} u}{\partial t^{2}}=0 \\
4 u+\frac{\partial^{2} u}{\partial y^{2}}+\frac{1}{12} \frac{\partial^{4} u}{\partial y^{4}}+\frac{\partial^{2} u}{\partial t^{2}}=0
\end{gathered}
$$

For the case of time-harmonic waves of the form

$$
u=A \exp (-i k y) \exp (i \omega t)
$$

equations (14) and (15) result in the following dispersion relations

for periodic mode

$$
\omega^{2}=k^{2}-\frac{1}{12} k^{4}
$$

for anti-periodic mode

$$
\omega^{2}=4-k^{2}+\frac{1}{12} k^{4}
$$

where $k=K l=2 \pi \varepsilon ; K$ is the dimensional wave number, $K=2 \pi / L$. It is clearly seen that expressions (17) and (18) coincide with the Taylor series expansions of the exact dispersion relations (6) and (7) as $k \rightarrow 0$ within the error $O\left(k^{6}\right)$.

\subsection{Macroscopic model}

Now we wish to derive a continuous model that can be applied within the entire region $0 \leq k \leq \pi$. Let us begin by considering a periodic mode. As $k \rightarrow 0$, the asymptotic approximation of the dispersion curve is given by expansion (17). In the opposite limit, $k \rightarrow \pi$, the asymptotic 
solution can be obtained from expression (18) by a substitution $k \Rightarrow \pi-k$. In order to reduce the order of the resulting macroscopic differential equation, we have to truncate series (17), (18). This is important so that to avoid additional boundary conditions requiring for the solution of macroscopic problems. Let us adopt the expansions

$$
\begin{gathered}
\omega^{2} \sim \omega_{02}^{2} k^{2} \quad \text { as } \quad k \rightarrow 0, \\
\omega^{2} \sim \omega_{\pi 0}^{2}+\omega_{\pi 2}^{2}(\pi-k)^{2} \quad \text { as } \quad k \rightarrow \pi .
\end{gathered}
$$

Here, for the monatomic lattice, $\omega_{02}^{2}=1, \omega_{\pi 0}^{2}=4, \omega_{\pi 2}^{2}=-1$.

We match expressions (19) and (20) using the method of two-point Padé approximants (Baker and Graves-Morris, 1985) and obtain:

$$
\omega^{2}=\frac{a_{1} k^{2}+a_{2} k^{4}}{1+a_{3} k^{2}+a_{4} k^{4}}
$$

Here the coefficients $a_{1}, \ldots, a_{4}$ are determined in such a way that the leading terms of the power series expansions of Padé approximant (21) at $k \rightarrow 0$ and $k \rightarrow \pi$ must coincide with expressions (19) and (20) up to $O\left(k^{2}\right), O\left[(\pi-k)^{2}\right]$ accordingly. Fulfilling this condition, we derive

$$
a_{1}=\omega_{02}^{2}, \quad a_{2}=-\frac{\pi^{4} \omega_{02}^{2} \omega_{\pi 2}^{2}+4 \omega_{\pi 0}^{4}}{\pi^{6} \omega_{\pi 2}^{2}}, \quad a_{3}=\frac{\pi^{2} \omega_{02}^{2}-2 \omega_{\pi 0}^{2}}{\pi^{2} \omega_{\pi 0}^{2}}, \quad a_{4}=\frac{\pi^{2} \omega_{\pi 2}^{2}\left(\omega_{\pi 0}^{2}-\pi^{2} \omega_{02}^{2}\right)-4 \omega_{\pi 0}^{4}}{\pi^{6} \omega_{\pi 0}^{2} \omega_{\pi 2}^{2}} .
$$

For the monatomic lattice, expressions (22) yield:

$$
a_{1}=1, \quad a_{2}=\frac{64-\pi^{4}}{\pi^{6}}, \quad a_{3}=\frac{\pi^{2}-8}{4 \pi^{2}}, \quad a_{4}=\frac{64+4 \pi^{2}-\pi^{4}}{4 \pi^{6}}
$$

In Fig. 4, formula (21) is compared with the exact dispersion relation (6). We note that within the pass band $(0 \leq \omega \leq 2)$ the numerical results are essentially indistinguishable. In the stop band (for $\omega>2$ ) the derived approximation provides a good asymptotic accuracy as $\omega \rightarrow 2+0$. 
$\omega$

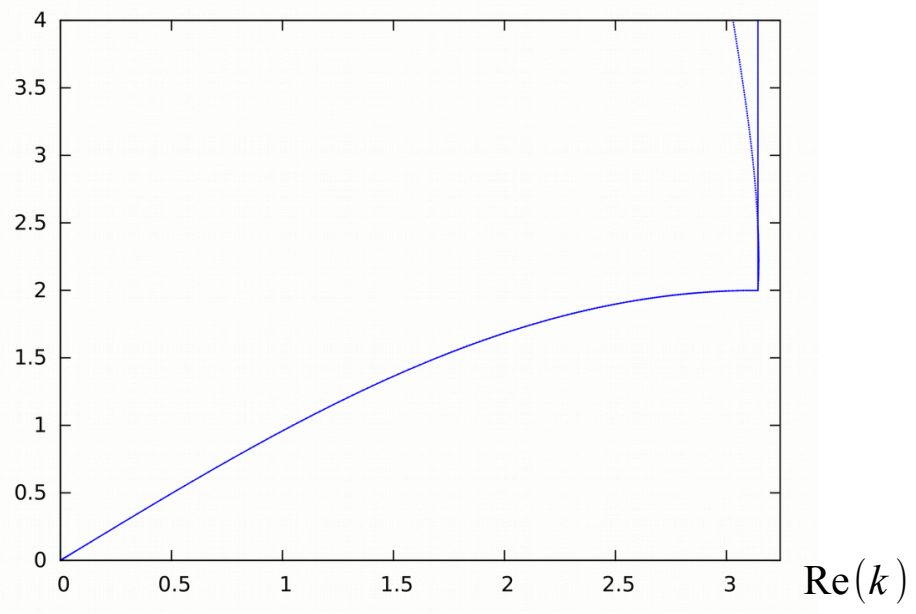

a) dispersion curve

$\omega$

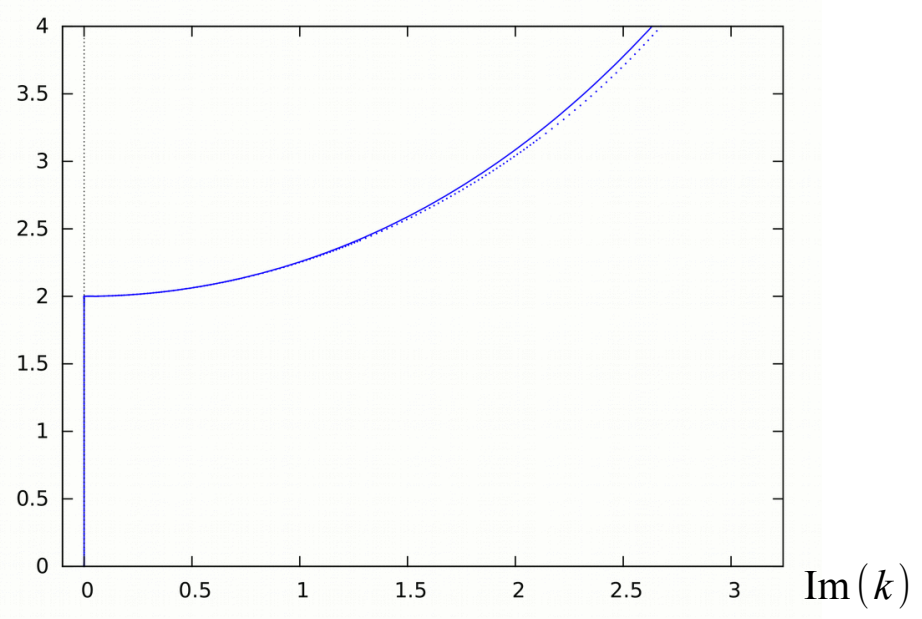

b) attenuation coefficient

Fig. 4. Dispersion diagram of the periodic mode in the monatomic lattice.

Solid curves - exact solution (6), dotted curves - Padé approximant (21).

The denominator of expression (21) does not equal zero for any real $k$. Therefore, formula (21) can be rewritten as follows

$$
\omega^{2}-a_{1} k^{2}-a_{2} k^{4}+a_{3} \omega^{2} k^{2}+a_{4} \omega^{2} k^{4}=0 .
$$

If the solution is given in the form of a harmonic wave (16), then

$$
\frac{\partial^{2} u}{\partial y^{2}}=-k^{2} u, \frac{\partial^{4} u}{\partial y^{4}}=k^{4} u, \frac{\partial^{2} u}{\partial t^{2}}=-\omega^{2} u, \frac{\partial^{4} u}{\partial y^{2} \partial t^{2}}=\omega^{2} k^{2} u, \frac{\partial^{6} u}{\partial y^{4} \partial t^{2}}=-\omega^{2} k^{4} u \text {. }
$$


Making use of expressions (25), the dispersion relation (24) allows us to obtain a higher-order differential equation that describes propagation of the periodic mode in the entire region of the wave number $0 \leq k \leq \pi$ :

$$
a_{1} \frac{\partial^{2} u}{\partial y^{2}}-a_{2} \frac{\partial^{4} u}{\partial y^{4}}+a_{3} \frac{\partial^{4} u}{\partial y^{2} \partial t^{2}}-a_{4} \frac{\partial^{6} u}{\partial y^{4} \partial t^{2}}=\frac{\partial^{2} u}{\partial t^{2}}
$$

For the anti-periodic mode, the solution can be developed in a similar way.

Equation (26) includes three dispersive terms and may be considered as a generalisation of double-dispersive equations, which were used by many authors to simulate elastic waves in waveguides with a free lateral surface (see, for example, Samsonov (2001), Porubov (2003) and references therein). In the theory of waves in structured solids, double- and triple-dispersion equations can be obtained by imposing some additional internal degrees of freedom on the system (see, for example, a review by Berezovski et al., 2011). It should be noted that such non-local models usually include a number of phenomenological parameters, which for real materials remain unknown and are expected to be determined in a purely experimental way. At the same time, the approach presented in this paper allows one to evaluate all the coefficients of equation (28) theoretically basing on the information about the internal structure of the medium and its properties. The developed macroscopic model is able to describe the long-wave case and, at the same time, it is valid in a high frequency range in the vicinity of the stop-band threshold.

\subsection{Long-wave case}

Let us consider the dynamical behaviour of the lattice in the long-wave case. If the wavelength $L$ is larger than the distance $l$ between the particles, the obtained macroscopic model (26) is able to describe asymptotically the propagation of the both periodic and anti-periodic modes. To show this, it is convenient to apply a two-scale asymptotic procedure.

In terms of the dimensional variable $X$, equation (26) reads

$$
a_{1} l^{2} \frac{\partial^{2} u}{\partial X^{2}}-\frac{\partial^{2} u}{\partial t^{2}}-a_{2} l^{4} \frac{\partial^{4} u}{\partial X^{4}}+a_{3} l^{2} \frac{\partial^{4} u}{\partial X^{2} \partial t^{2}}-a_{4} l^{4} \frac{\partial^{6} u}{\partial X^{4} \partial t^{2}}=0
$$

Instead of the original co-ordinate $X$, let us introduce so called slow $x$ and fast $y$ 
variables:

$$
x=X / L, y=X / l \text {. }
$$

The slow co-ordinate is used for study of the problem on macro scale, which is associated with the wavelength. The fast co-ordinate is intended to describe the lattice dynamics on micro scale within a distinguished unit cell. The spatial derivatives are given by

$$
\begin{gathered}
\frac{\partial^{2}}{\partial X^{2}}=\frac{1}{L^{2}}\left(\frac{\partial^{2}}{\partial x^{2}}+2 \varepsilon^{-1} \frac{\partial^{2}}{\partial x \partial y}+\varepsilon^{-2} \frac{\partial^{2}}{\partial y^{2}}\right) \\
\frac{\partial^{4}}{\partial X^{4}}=\frac{1}{L^{4}}\left(\frac{\partial^{4}}{\partial x^{4}}+4 \varepsilon^{-1} \frac{\partial^{4}}{\partial x^{3} \partial y}+6 \varepsilon^{-2} \frac{\partial^{4}}{\partial x^{2} y^{2}}+4 \varepsilon^{-3} \frac{\partial^{4}}{\partial x \partial y^{3}}+\varepsilon^{-4} \frac{\partial^{4}}{\partial y^{4}}\right)
\end{gathered}
$$

where $\varepsilon$ is the small parameter, $\varepsilon=l / L$. Then equation (27) reads

$$
\begin{gathered}
\left(a_{1}+a_{3} \frac{\partial^{2}}{\partial t^{2}}\right)\left(\frac{\partial^{2} u}{\partial y^{2}}+2 \varepsilon \frac{\partial^{2} u}{\partial x \partial y}+\varepsilon^{2} \frac{\partial^{2} u}{\partial x^{2}}\right)- \\
\left(a_{2}+a_{4} \frac{\partial^{2}}{\partial t^{2}}\right)\left(\frac{\partial^{4} u}{\partial y^{4}}+4 \varepsilon \frac{\partial^{4} u}{\partial x \partial y^{3}}+6 \varepsilon^{2} \frac{\partial^{4} u}{\partial x^{2} y^{2}}+4 \varepsilon^{3} \frac{\partial^{4} u}{\partial x^{3} \partial y}+\varepsilon^{4} \frac{\partial^{4} u}{\partial x^{4}}\right)-\frac{\partial^{2} u}{\partial t^{2}}=0
\end{gathered}
$$

Considering propagation of a harmonic wave, the displacement field can be represented as follows $u(x, t)=w(x) \exp (i \omega t)$. We shall search for a solution in the form of asymptotic expansions

$$
\begin{aligned}
& w=w_{0}+w_{1} \varepsilon+w_{2} \varepsilon^{2}+\ldots, \\
& \omega^{2}=\omega_{0}^{2}+\omega_{1}^{2} \varepsilon+\omega_{2}^{2} \varepsilon^{2}+\ldots
\end{aligned}
$$

Splitting equation (29) with respect to $\varepsilon$, in the leading order approximation we obtain

$$
\omega_{0}^{2} w_{0}+\left(a_{1}-a_{3} \omega_{0}^{2}\right) \frac{\partial^{2} w_{0}}{\partial y^{2}}-\left(a_{2}-a_{4} \omega_{0}^{2}\right) \frac{\partial^{4} w_{0}}{\partial y^{4}}=0
$$

Separating slow and fast components of the displacement field, we can represent $w_{0}$ as follows: 


$$
w_{0}(x, y)=W(y) F(x) .
$$

Here $W(y)$ describes a local solution across the unite cell, whereas $F(x)$ accounts for the behaviour of the lattice on macro scale. Substituting (33) into (32), we derive

- for periodic mode: $\quad W(y)=A, \quad \omega_{0}^{2}=0$;

- for anti-periodic mode: $\quad W(y)=A \exp (i \pi y), \quad \omega_{0}^{2}=\frac{a_{1} \pi^{2}+a_{2} \pi^{4}}{1+a_{3} \pi^{2}+a_{4} \pi^{4}}=\omega_{\pi 0}^{2}$;

where $A$ is the constant amplitude.

In the first order approximation equation (29) gives

$$
\begin{gathered}
\omega_{0}{ }^{2} w_{1}+\left(a_{1}-a_{3} \omega_{0}{ }^{2}\right) \frac{\partial^{2} w_{1}}{\partial y^{2}}-\left(a_{2}-a_{4} \omega_{0}{ }^{2}\right) \frac{\partial^{4} w_{1}}{\partial y^{4}}= \\
-\omega_{1}^{2} w_{0}-2\left(a_{1}-a_{3} \omega_{0}^{2}\right) \frac{\partial^{2} w_{0}}{\partial x \partial y}+a_{3} \omega_{1}^{2} \frac{\partial^{2} w_{0}}{\partial y^{2}}+4\left(a_{2}-a_{4} \omega_{0}^{2}\right) \frac{\partial^{4} w_{0}}{\partial x \partial y^{3}}-a_{4} \omega_{1}^{2} \frac{\partial^{4} w_{0}}{\partial y^{2}}
\end{gathered}
$$

A straightforward integration of equation (34) will lead to secular terms in the expression for $w_{1}$. To avoid them, in the r.h.s. of equation (34) the coefficients of the terms which are a part of the homogeneous solution must be equal to zero. Thus, we must set to zero the coefficients at $A F(x)$ (for periodic mode) and at $A \exp (i \pi y) F(x)$ (for anti-periodic mode).

Fulfilling the aforementioned conditions, we obtain equations that relate $\omega_{1}^{2}$ and $F(x)$ :

- for periodic mode:

$$
\begin{gathered}
\omega_{1}^{2} F(x)=0, \\
\omega_{1}^{2}\left(1+a_{3} \pi^{2}+a_{4} \pi^{4}\right) F(x)=0 .
\end{gathered}
$$$$
\text { - for anti-periodic mode: }
$$

For any non-zero $F(x)$ we get $\omega_{1}^{2}=0$ for the both periodic and anti-periodic modes. Then, for the given values of the coefficients $a_{1}, \ldots, a_{4}$ (25), the r.h.s. of equation (34) vanishes and we derive $w_{1}$ in the form similar to $w_{0}$ :

- for periodic mode:

$$
\begin{array}{lc}
\text { - for periodic mode: } & w_{1}=A F(x), \\
\text { - for anti-periodic mode: } & w_{1}=A \exp (i \pi y) F(x) .
\end{array}
$$


In the second order approximation equation (29) gives

$$
\begin{gathered}
\omega_{0}{ }^{2} w_{2}+\left(a_{1}-a_{3} \omega_{0}^{2}\right) \frac{\partial^{2} w_{2}}{\partial y^{2}}-\left(a_{2}-a_{4} \omega_{0}^{2}\right) \frac{\partial^{4} w_{2}}{\partial y^{4}}=-\omega_{2}^{2} w_{0}-\left(a_{1}-a_{3} \omega_{0}^{2}\right)\left(\frac{\partial^{2} w_{0}}{\partial x^{2}}+2 \frac{\partial^{2} w_{1}}{\partial x \partial y}\right)+ \\
\left(a_{2}-a_{4} \omega_{0}^{2}\right)\left(6 \frac{\partial^{4} w_{0}}{\partial x^{2} \partial y^{2}}+4 \frac{\partial^{4} w_{1}}{\partial x \partial y^{3}}\right)+a_{3} \omega_{2}^{2} \frac{\partial^{2} w_{0}}{\partial y^{2}}-a_{4} \omega_{2}^{2} \frac{\partial^{4} w_{0}}{\partial y^{4}}
\end{gathered}
$$

Applying to the r.h.s. of equation (35) the condition of elimination of secular terms, we obtain

$$
\text { for periodic mode: } \quad a_{1} \frac{d^{2} F(x)}{d x}+\omega_{2}^{2} F(x)=0
$$

for anti-periodic mode: $\left(a_{1}+6 a_{2} \pi^{2}-a_{3} \omega_{0}^{2}-6 a_{4} \pi^{2} \omega_{0}^{2}\right) \frac{d^{2} F(x)}{d x}+\omega_{2}^{2}\left(1+a_{3} \pi^{2}+a_{4} \pi^{4}\right) F(x)=0$.

In a general case, the macroscopic wave filed $F(x)$ may be determined from a boundary value problem formulated for the entire lattice on macro level. Knowing $F(x)$, it becomes possible to evaluate the next coefficient $\omega_{2}^{2}$ of the frequency expansion. Here we consider a propagation of harmonic waves and accept

$$
F(x)=\exp (-i K X)=\exp (-i 2 \pi x)
$$

Then equations (36), (37) give: $\omega_{2}^{2}=4 \pi^{2} \omega_{02}^{2}$ (for periodic mode) and $\omega_{2}^{2}=4 \pi^{2} \omega_{\pi 2}^{2}$ (for antiperiodic mode).

Taking into account $\varepsilon=k /(2 \pi), k \rightarrow 0$, the asymptotic formulas for the dispersion relation eventually read

$$
\begin{array}{lc}
\text { for periodic mode: } & \bar{\omega}^{2} \sim \omega_{02}^{2} k^{2}, \\
\text { for anti-periodic mode: } & \bar{\omega}^{2} \sim \omega_{\pi 0}^{2}+\omega_{\pi 2}^{2} k^{2} .
\end{array}
$$

Expressions (39), (40) coincide with expansions (17), (18) of the exact solution up to $O\left(k^{2}\right)$.

In Fig. 5, the asymptotic formulas (39), (40) are compared with the exact dispersion relations (6), (7). We may conclude that the derived macroscopic equation (26) provides a good numerical accuracy for the both periodic and anti-periodic modes when the wave length is at least 
five-six times longer than the size of the microstructure.

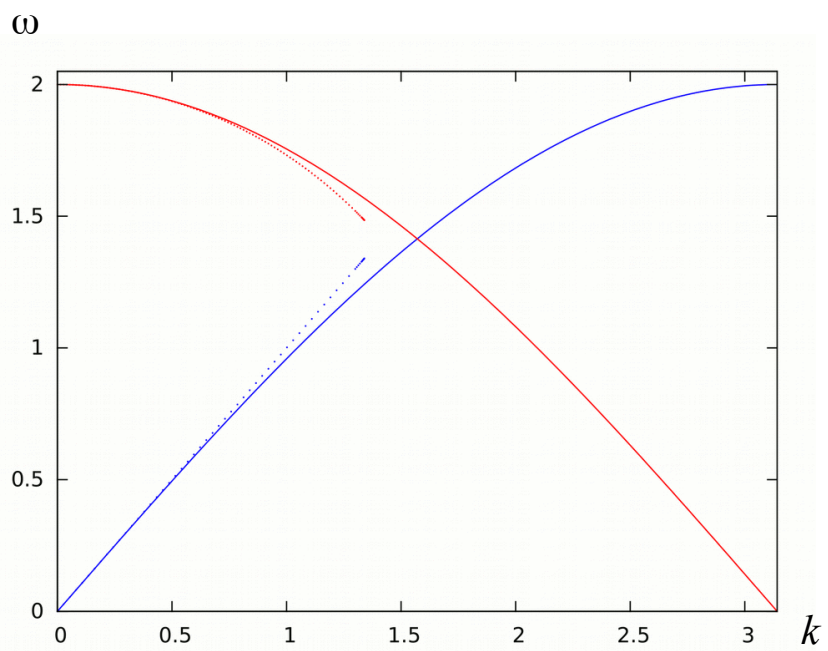

Fig. 5. Dispersion curves for periodic (blue) and anti-periodic (red) modes.

Solids - exact solutions (6), (7); dots - asymptotic formulas (39), (40).

We have shown that the macroscopic model (26) captures the dispersive properties of the lattice. Moreover, it can asymptotically reproduce the structure of the original macroscopic equations (12), (13). Following the two-scale asymptotic procedure, let us represent the displacement field as $u=W(y) \widetilde{u}(x, t)$. Here $W(y)$ accounts for the local solution within the unit cell and $\widetilde{u}(x, t)$ is the effective wave field describing propagation of the envelope wave.

For the periodic mode $W=A$. Then equation (29) gives

$$
a_{1} \varepsilon^{2} \frac{\partial^{2} \tilde{u}}{\partial x^{2}}-\frac{\partial^{2} \tilde{u}}{\partial t^{2}}-a_{2} \varepsilon^{4} \frac{\partial^{4} \tilde{u}}{\partial x^{4}}+a_{3} \varepsilon^{2} \frac{\partial^{4} \tilde{u}}{\partial x^{2} \partial t^{2}}-a_{4} \varepsilon^{4} \frac{\partial^{6} \tilde{u}}{\partial x^{4} \partial t^{2}}=0
$$

Within the error $O\left(\varepsilon^{4}\right)$, expression (41) can be transformed as follows

$$
\frac{\partial^{2} \tilde{u}}{\partial t^{2}}=\frac{a_{1} \varepsilon^{2} \frac{\partial^{2} \tilde{u}}{\partial x^{2}}}{1-a_{3} \varepsilon^{2} \frac{\partial^{2}}{\partial x^{2}}}=a_{1} \varepsilon^{2} \frac{\partial^{2} \tilde{u}}{\partial x^{2}}
$$

For the monatomic lattice $a_{1}=1$. We observe that equations (42) and (12) coincide up to $O\left(\varepsilon^{2}\right)$.

In the anti-periodic case $W=A \exp (i \pi y)$. Splitting equation (29) with respect to $\varepsilon$, we derive a sequence of constitutive equations of various orders. The leading order approximation is 


$$
\left(a_{1} \pi^{2}+a_{2} \pi^{4}\right) \widetilde{u}+\left(1+a_{3} \pi^{2}+a_{4} \pi^{4}\right) \frac{\partial^{2} \widetilde{u}}{\partial t^{2}}=0
$$

Taking into account the magnitudes of the coefficients $a_{1}, \ldots, a_{4}$ (23), expression (43) reads

$$
\frac{16}{\pi^{2}}\left(4 \tilde{u}+\frac{\partial^{2} \tilde{u}}{\partial t^{2}}\right)=0
$$

In the first order approximation we obtain

$$
\left[\left(a_{1}+2 a_{2} \pi^{2}\right) \frac{\partial \tilde{u}}{\partial x}+\left(a_{3}+2 a_{4} \pi^{2}\right) \frac{\partial^{3} \tilde{u}}{\partial x t^{2}}\right] \varepsilon,
$$

which for the monatomic lattice takes the form

$$
\frac{128-\pi^{4}}{\pi^{4}} \frac{\partial}{\partial x}\left(4 \widetilde{u}+\frac{\partial^{2} \widetilde{u}}{\partial t^{2}}\right) \varepsilon
$$

We note that in the formula above the expression in brackets is of order $O\left(\varepsilon^{2}\right)$. Therefore, expression (45) vanishes up to $O\left(\varepsilon^{3}\right)$.

The second order approximation reads

$$
\left[\left(a_{1}+6 a_{2} \pi^{2}\right) \frac{\partial^{2} \tilde{u}}{\partial x^{2}}+\left(a_{3}+6 a_{4} \pi^{2}\right) \frac{\partial^{4} \tilde{u}}{\partial x^{2} t^{2}}\right] \varepsilon^{2} .
$$

Substituting here the magnitudes of $a_{1}, \ldots, a_{4}$ (23), we obtain

$$
\frac{5 \pi^{4}-384}{\pi^{4}} \frac{\partial^{2}}{\partial x^{2}}\left(4 \widetilde{u}+\frac{\partial^{2} \tilde{u}}{\partial t^{2}}\right) \varepsilon^{2}-\frac{4}{\pi^{2}} \frac{\partial^{4} \tilde{u}}{\partial x^{2} \partial t^{2}} \varepsilon^{2}
$$

Here the expression in brackets is of order $O\left(\varepsilon^{2}\right)$, therefore, the first term in (46) can be neglected up to $O\left(\varepsilon^{4}\right)$. Making use of equation (44), the second term in (46) gives 


$$
\frac{16}{\pi^{2}} \frac{\partial^{2} \tilde{u}}{\partial x^{2}} \varepsilon^{2}
$$

Finally, combining expressions (44), (45) and (47), we obtain the macroscopic equation of motion as follows

$$
4 \widetilde{u}+\frac{\partial^{2} \tilde{u}}{\partial t^{2}}+\frac{\partial^{2} \tilde{u}}{\partial x^{2}} \varepsilon^{2}=0
$$

We can conclude that equations (48) and (13) coincide up to $O\left(\varepsilon^{2}\right)$.

\subsection{Dynamic response to the external loads}

The derived macroscopic model (26) can be applied to solving boundary value problems. As a benchmark test, let us study the dynamic response of the semi-infinite ( $y \geq 0)$ monatomic lattice to an external load applied at the edge $y=0$. We consider two types of excitations: a pulse load and a harmonic load.

The important feature of the pulse load problem is that during the transient wave propagation the coupling forces between the particles can exceed sufficiently the magnitude of the initial excitation. This effect is caused by a spatial redistribution of energy due to the heterogeneity of the structure and it can never be observed in homogeneous media. One of the earliest studies of the subject was presented by Filimonov et al. (1991). Some recent results can found, for example, in papers by Metrikine (2006), Askes et al. (2008), Andrianov et al. (2012) and references therein.

In the non-dimensional form, the governing dynamic model is given by equation (26). The initial and the boundary conditions read

$$
\begin{gathered}
\left.u\right|_{t=0}=\left.\frac{\partial u}{\partial t}\right|_{t=0}=0 \\
a_{1} \frac{\partial u}{\partial y}-a_{2} \frac{\partial^{3} u}{\partial y^{3}}+a_{3} \frac{\partial^{3} u}{\partial y \partial t^{2}}-\left.a_{4} \frac{\partial^{5} u}{\partial y^{3} \partial t^{2}}\right|_{y=0}=-p_{0}(t) l \\
u \rightarrow 0 \text { as } y \rightarrow \infty
\end{gathered}
$$

where the function $p_{0}(t)$ describes the external excitation. The boundary condition (50) is obtained 
through the first spatial integration of the 1.h.s. of equation (26), which is associated with a time dependent elastic force. For the pulse load $p_{0}(t)=p \delta(t)$ and for the harmonic load $p_{0}(t)=p \sin (\omega t)$. Here $p=P /(c l) ; P$ is the amplitude of the external force; $\delta(t)$ is the Dirac delta function.

Boundary conditions (50), (51) come naturally from the physical reasons. It should be noted that equation (26) includes the fourth-order spatial derivatives and, consequently, additional boundary conditions are required. This is a typical difficulty that arises when higher-order models, derived originally for infinite media, are applied to bounded domains. Kaplunov and Pichugin (2009) have shown that general solutions of the higher-order models combine contributions of long wave solutions associated with the macroscopic problem and short wave solutions localised in the vicinity of boundaries. The latter are induced particularly by the presence of higher-order derivative terms. The short wave solutions describe extraneous boundary layers that can be considered as artefacts of the approximated model. Therefore, additional boundary conditions for equation (26) should be formulated in such a way to eliminate spurious short wave solutions. This principle yields (Kaplunov and Pichugin, 2009):

$$
\left.\frac{\partial^{2} u}{\partial y^{2}}\right|_{y=0}=0
$$

Problem (26), (49)-(52) can be solved by applying the Laplace transform

$$
u_{s}(y, s)=\int_{0}^{\infty} u(y, t) \exp (-s t) d t
$$

In the Laplace domain we obtain

$$
\begin{gathered}
-s^{2} u_{s}+\left(a_{1}+s^{2} a_{3}\right) \frac{\partial^{2} u_{s}}{\partial y^{2}}-\left(a_{2}+s^{2} a_{4}\right) \frac{\partial^{4} u_{s}}{\partial y^{4}}=0 \\
\left(a_{1}+s^{2} a_{3}\right) \frac{\partial u_{s}}{\partial y}-\left.\left(a_{2}+s^{2} a_{4}\right) \frac{\partial^{3} u_{s}}{\partial y^{3}}\right|_{y=0}=-p l \text { for the pulse load, } \\
\left(a_{1}+s^{2} a_{3}\right) \frac{\partial u_{s}}{\partial y}-\left.\left(a_{2}+s^{2} a_{4}\right) \frac{\partial^{3} u_{s}}{\partial y^{3}}\right|_{y=0}=-p l \frac{\omega}{s^{2}+\omega^{2}} \text { for the harmonic load; }
\end{gathered}
$$




$$
\begin{gathered}
\left.\frac{\partial^{2} u_{s}}{\partial y^{2}}\right|_{y=0}=0 ; \\
u_{s} \rightarrow 0 \text { as } y \rightarrow \infty .
\end{gathered}
$$

A general solution of equation (53) that meets the boundary condition (56) read

$$
u_{s}=C_{1} \exp \left(-\kappa_{1} y\right)+C_{2} \exp \left(-\kappa_{2} y\right) \text {. }
$$

Substituting (57) into (53), we get a characteristic equation for $\kappa_{1}, \kappa_{2}$ as follows

$$
\left(a_{2}+s^{2} a_{4}\right) \kappa^{4}-\left(a_{1}+s^{2} a_{3}\right) \kappa^{2}+s^{2}=0 .
$$

In order to implement condition (56), we have to choose two positive roots of equation (58) such that $\operatorname{Re}\left(\kappa_{1}, \kappa_{2}\right)>0$ for $\operatorname{Re}(s)>0$. Then we obtain

$$
\kappa_{1,2}=\sqrt{\frac{a_{1}+s^{2} a_{3} \pm \sqrt{s^{4}\left(a_{3}^{2}-4 a_{4}\right)+2 s^{2}\left(a_{1} a_{3}-2 a_{2}\right)+a_{1}^{2}}}{2\left(a_{2}+s^{2} a_{4}\right)}} .
$$

Substituting (57), (59) into the boundary conditions (54), (55) provides us with a system of two linear equations for the constants $C_{1}, C_{2}$, which yields for the pulse load

$$
C_{1}=\frac{\kappa_{2} p l}{D_{1}+s^{2} D_{2}}, \quad C_{2}=-\frac{\kappa_{1}^{2}}{\kappa_{2}^{2}} C_{1} ;
$$

and for the harmonic one

$$
C_{1}=\frac{\kappa_{2} p l \omega}{\left(D_{1}+s^{2} D_{2}\right)\left(s^{2}+\bar{\omega}^{2}\right)}, \quad C_{2}=-\frac{\kappa_{1}^{2}}{\kappa_{2}^{2}} C_{1}
$$

where $D_{1}=-a_{1} \kappa_{1}^{2}+\left(a_{1}-a_{2} \kappa_{1}^{2}\right) \kappa_{1} \kappa_{2}+a_{2} \kappa_{1}^{2} \kappa_{2}^{2}, D_{2}=-a_{3} \kappa_{1}^{2}+\left(a_{3}-a_{4} \kappa_{1}^{2}\right) \kappa_{1} \kappa_{2}+a_{4} \kappa_{1}^{2} \kappa_{2}^{2}$.

The inverse Laplace transform is determined by the integral 


$$
u(y, t)=\frac{1}{2 \pi i} \int_{\gamma-i \infty}^{\gamma+i \infty} u_{s}(y, s) \exp (s t) d s
$$

Here $\gamma$ is a real positive constant that has to be larger than the real parts of all singularities of the function $u_{s}$. Since the derived solution has no singularities at the right half-plane of the complex $s$ plane, we may choose any positive value of $\gamma$. In the examples below integral (60) is evaluated numerically in Maple with $\gamma=0.1$.

Numerical results for the case of the pulse load are given at Fig. 6. The displacement field is presented at two successive moments of time for $p=1$. The obtained analytical solution is verified by the results of the direct numerical simulation performed by the Runge-Kutta fourth-order method. For a comparison, we also display the transient wave profile for the homogeneous (i.e., non-dispersive) continuum model. The macroscopic model (26) demonstrates a high numerical accuracy.

It should be noted that the profile of the transient waves in the discrete lattice is qualitatively similar to the wave fronts appearing in plates and shells (see, for example, Kaplunov et al. (2000) and references therein). This displays an analogue between the propagation of elastic waves in heterogeneous media and in thin waveguides (Craster et al., 2014).

In order to justify the choice of the higher-order boundary condition, two additional solutions are evaluated, when instead of equation (52) we assume $\partial u /\left.\partial y\right|_{y=0}=0$ or $\partial^{3} u /\left.\partial y^{3}\right|_{y=0}=0$. The obtained numerical results are shown at Fig. 7. Comparing Fig. $6 b$ and Fig. 7, one can observe that equation (52) ensures the best practical accuracy.

The dynamic response of the lattice subjected to the harmonic load is displayed at Fig. 8 . The displacement field is evaluated for $t=4 \pi$ and $p=1$ at different values of the excitation frequency $\omega$. At a relatively low frequency $\omega=0.5$, which corresponds to the nearly straight part of the dispersion curve (see Fig. $4 a$ ), the behaviour of the lattice can be approximated by the non-dispersive model. With the increase of $\omega$ the solution differs significantly form the non-dispersive case. The macroscopic model (26) is applicable in the entire frequency range up to the value $\omega=2$, which corresponds to the stop-band threshold. 


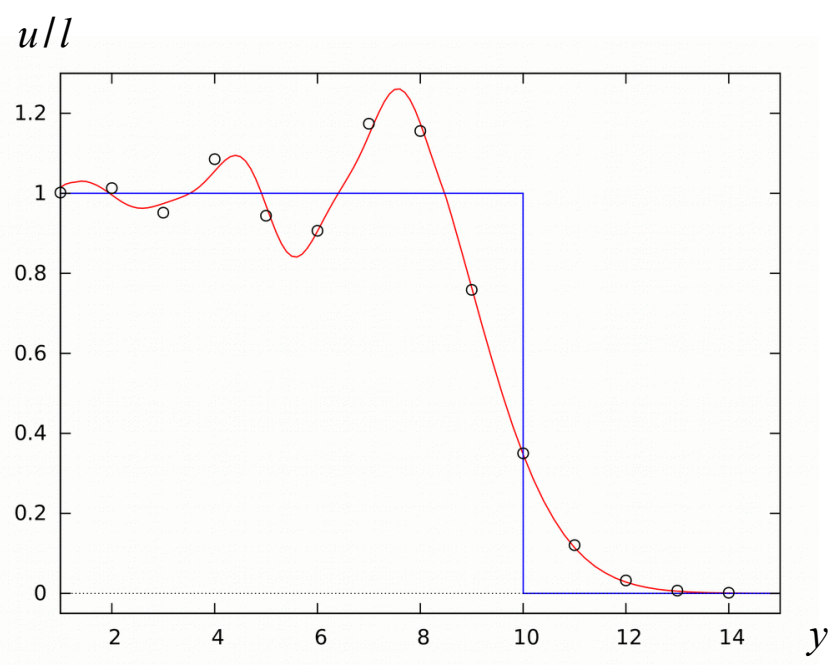

a) $t=10$

$u / l$

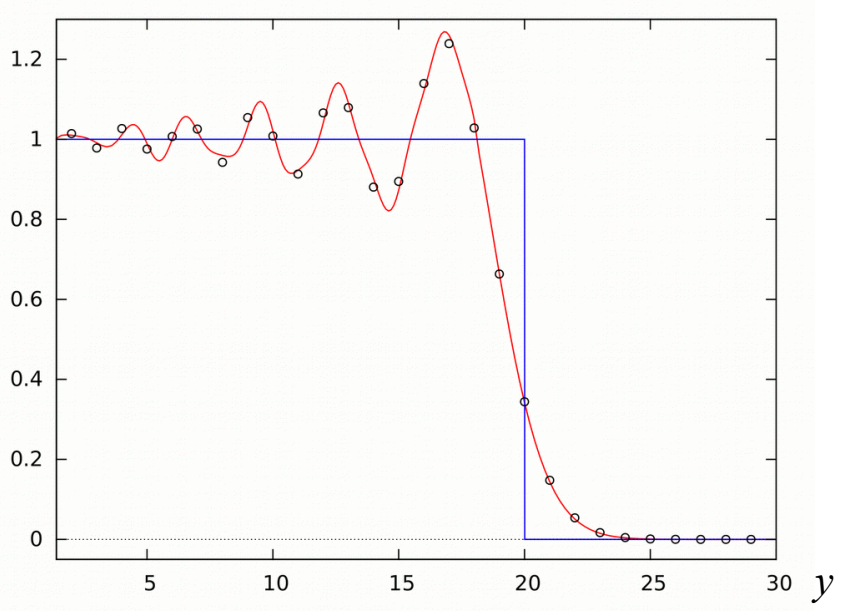

b) $t=20$

Fig. 6. Dynamic response of the monatomic lattice to the pulse load. Red - analytical solution (57); blue - non-dispersive solution; dots - data of the numerical simulation. 


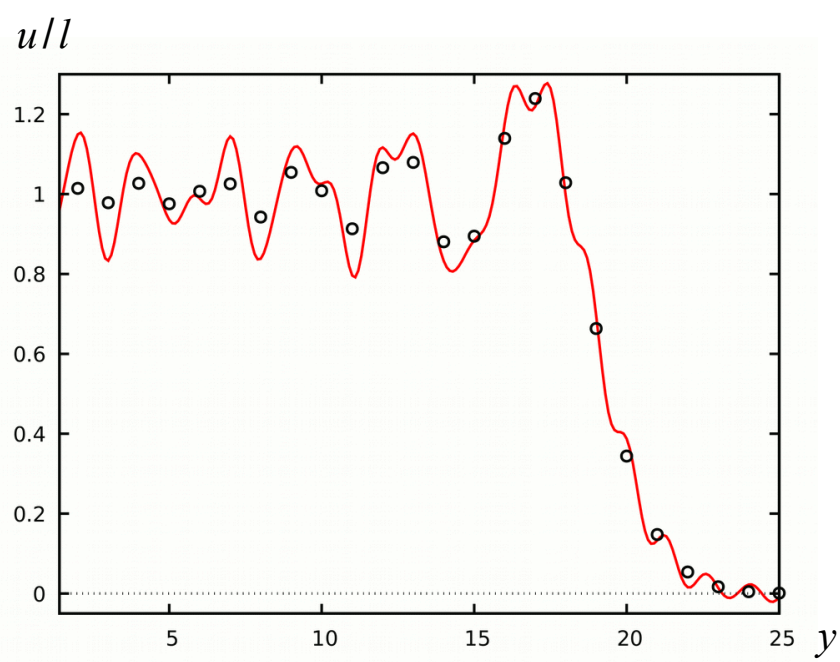

a) $\partial u /\left.\partial y\right|_{y=0}=0$

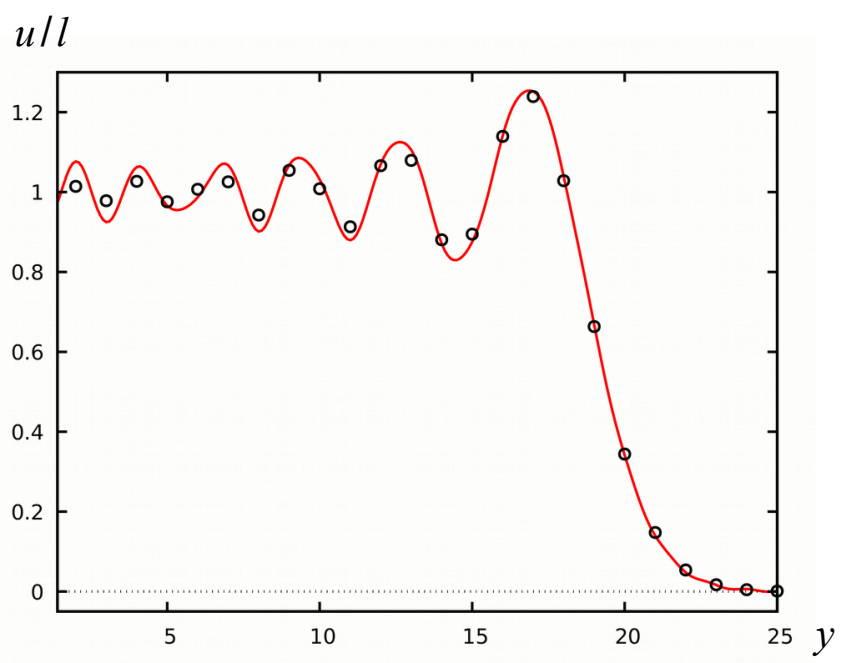

b) $\partial^{3} u /\left.\partial y^{3}\right|_{y=0}=0$

Fig. 7. Dynamic response to the pulse load evaluated with different boundary conditions; $t=20$. Red - analytical solution (57); dots - data of the numerical simulation. 


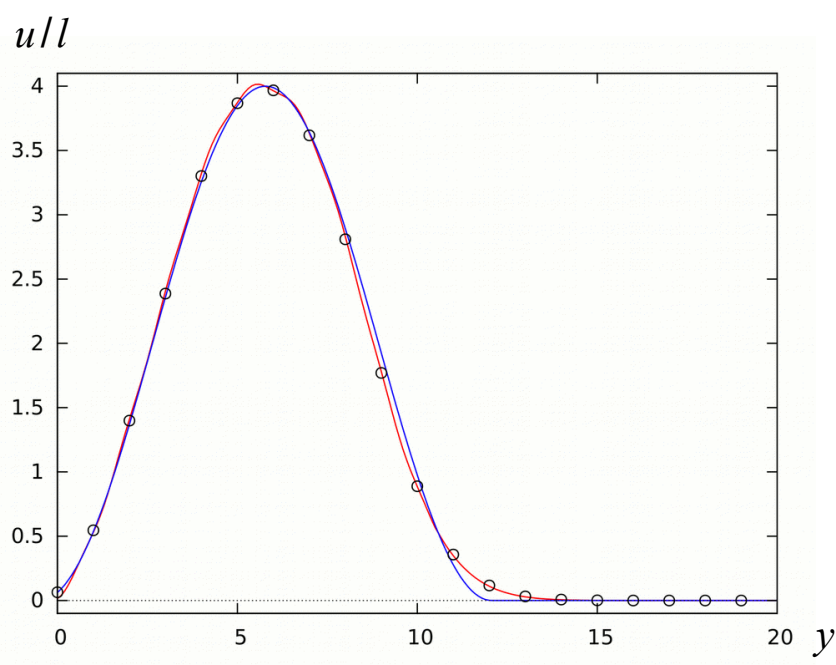

a) $\omega=0.5$

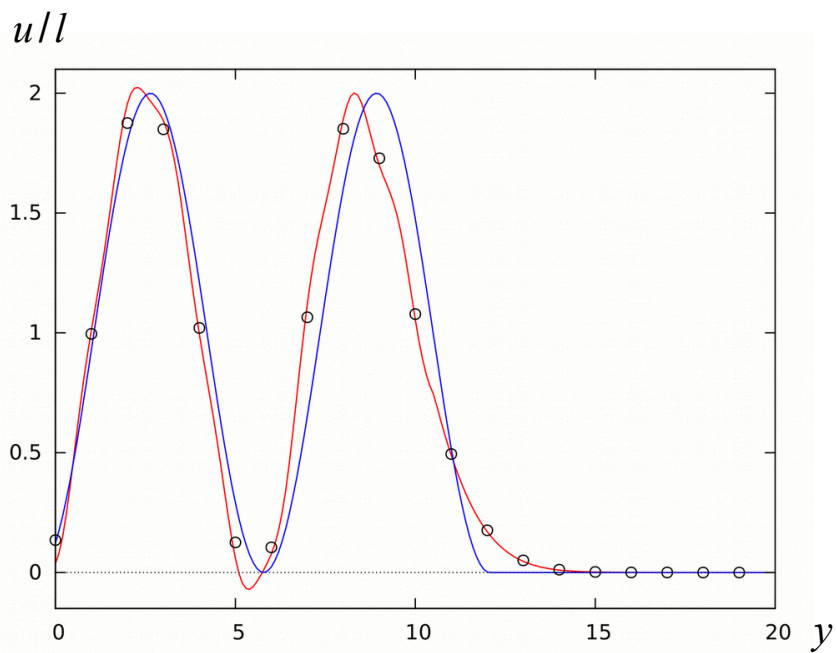

b) $\omega=1$

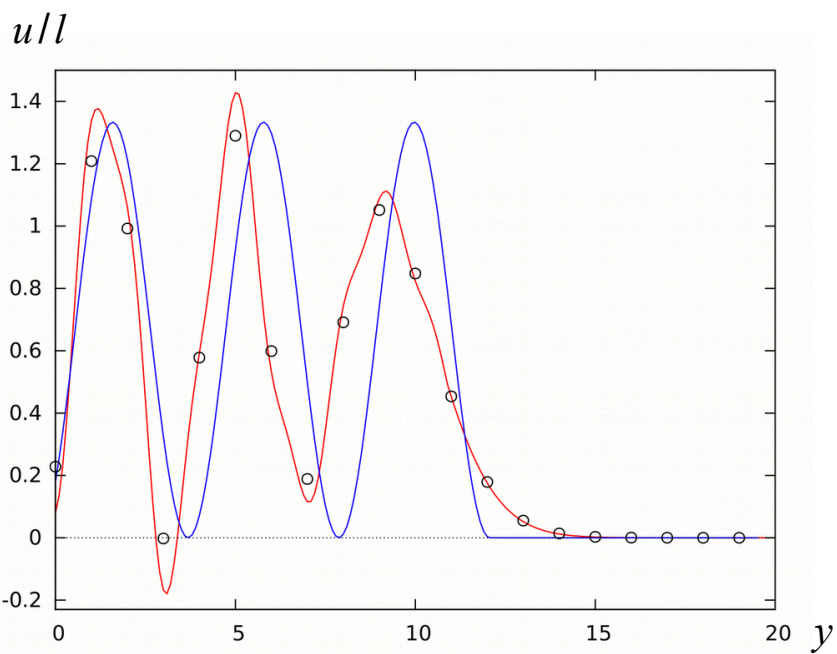

c) $\omega=1.5$ 


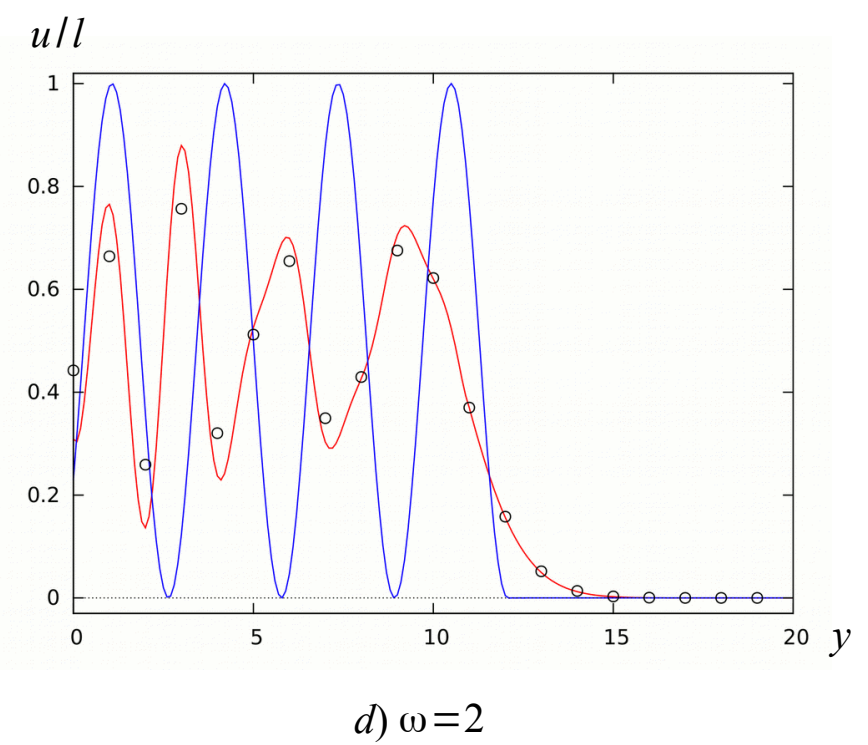

Fig. 8. Dynamic response of the monatomic lattice to the harmonic load. Red - analytical solution (57); blue - non-dispersive solution; dots - data of the numerical simulation.

\section{Periodically heterogeneous waveguides}

\subsection{One-dimensional periodic media}

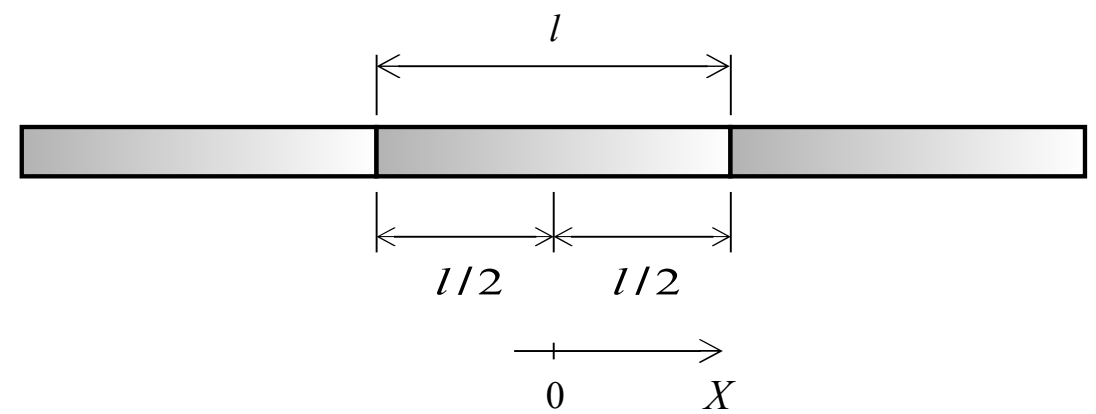

Fig. 9. Periodically heterogeneous medium.

Now we aim to extend the procedure developed above to continuous media. Let us consider propagation of one-dimensional waves in a heterogeneous solid consisting of periodically repeated unit cells of the length $l$ (Fig. 9). The governing wave equation reads

$$
\frac{\partial}{\partial X}\left[E(X) \frac{\partial u}{\partial X}\right]-\rho(X) \frac{\partial^{2} u}{\partial T^{2}}=0,
$$


where $E(X)$ and $\rho(X)$ are coordinate dependent physical properties of the medium, namely, the elastic constant and the mass density. We suppose $E(X)$ and $\rho(X)$ to be spatially periodic, $E(X)=E(X+l), \rho(X)=\rho(X+l)$, with a certain (in general, arbitrary) distribution within the unit cell.

Without loss of generality and for the reason of simplicity, let us assume $E(X)$ to be constant. Considering harmonic wave $u(X)=w(X, T) \exp (i \Omega T)$, equation (61) can be written as follows

$$
l^{2} \frac{d^{2} w}{d X^{2}}+\frac{\hat{v}^{2}}{v^{2}(X)} \omega^{2} w=0
$$

where $v(X)$ is the wave speed, $v^{2}(X)=E / \rho(X) ; \hat{v}$ is the homogenised wave speed, $\hat{v}=\int_{-l / 2}^{l / 2} v(X) d X ; \omega$ is the non-dimensional frequency, $\omega=\Omega l / \hat{v}$.

In order to develop asymptotic expansions of the dispersion curves, we apply the two-scale asymptotic procedure (Craster et al., 2010a). Making the change of variables (28), equation (62) gives

$$
\frac{\partial^{2} w}{\partial y^{2}}+\frac{\hat{v}^{2}}{v^{2}(y)} \omega^{2} w+2 \varepsilon \frac{\partial^{2} w}{\partial x \partial y}+\varepsilon^{2} \frac{\partial^{2} w}{\partial y^{2}}=0
$$

where $\varepsilon$ is the small parameter, $\varepsilon=l / L ; L$ is the wavelength.

We represent the solution as series expansions (30), (31) in powers of $\varepsilon$. In the leading order approximation, we obtain

$$
w_{0}(x, y)=W_{0}(y) F(x)
$$

where $W_{0}(y)$ is the local solution across the unit cell, $F(x)$ is the macroscopic wave field.

$W_{0}(y)$ can be determined from the equation

$$
\frac{d^{2} W_{0}}{d y^{2}}+\frac{\hat{v}^{2}}{v^{2}(y)} \omega_{0}^{2} W_{0}=0
$$


subject to the boundary conditions

$$
\left.W_{0}\right|_{y=-\frac{1}{2}}= \pm\left. W_{0}\right|_{y=\frac{1}{2}},\left.\quad \frac{d W_{0}}{d y}\right|_{y=-\frac{1}{2}}= \pm\left.\frac{d W_{0}}{d y}\right|_{y=\frac{1}{2}}
$$

where "+" accounts for periodic and "-" for anti-periodic modes.

The eigenvalue problem (64), (65) allows evaluation of the leading order term $\omega_{0}^{2}$ of the frequency expansion. It determines the edges of phononic bands. Since the continuous medium exhibits a sequence of pass and stop bands, an infinite number of eigenvalues $\omega_{0}^{2}$ can be derived.

In the first order approximation, equation (63) gives

$$
\frac{\partial^{2} w_{1}}{\partial y^{2}}+\frac{\hat{v}^{2}}{v^{2}(y)} \omega_{0}^{2} w_{1}=-2 \frac{\partial^{2} w_{0}}{\partial x \partial y}-\frac{\hat{v}^{2}}{v^{2}(y)} \omega_{1}^{2} w_{0} .
$$

In order to avoid secular terms, one has to multiply the r.h.s. of equation (66) by $w_{0}$, integrate over the periodic unit cell, and set the result to zero. Fulfilling this condition, we obtain $\omega_{1}^{2}=0$. Then $w_{1}$ is derived as follows

$$
w_{1}=\left[W_{1}(y)-y W_{0}(y)\right] \frac{F(x)}{d x}+W_{0}(y) F(x),
$$

where the function $W_{1}(y)$ is the solution of the leading-order equation

$$
\frac{d^{2} W_{1}}{d y^{2}}+\frac{\hat{v}^{2}}{v^{2}(y)} \omega_{0}^{2} W_{1}=0
$$

accompanied by the boundary conditions

$$
\left.w_{1}\right|_{y=-\frac{1}{2}}= \pm\left. w_{1}\right|_{y=\frac{1}{2}},\left.\quad \frac{d w_{1}}{d y}\right|_{y=-\frac{1}{2}}= \pm\left.\frac{d w_{1}}{d y}\right|_{y=\frac{1}{2}} .
$$

Here again "+" is for periodic and "-"” is for anti-periodic modes.

In the second order approximation we obtain 


$$
\frac{\partial^{2} w_{2}}{\partial y^{2}}+\frac{\hat{v}^{2}}{v^{2}(y)} \omega_{0}^{2} w_{2}=-\frac{\partial^{2} w_{0}}{\partial x^{2}}-2 \frac{\partial^{2} w_{1}}{\partial x \partial y}-\frac{\hat{v}^{2}}{v^{2}(y)} \omega_{2}^{2} w_{0}
$$

The condition of eliminating secular terms gives

$$
R \frac{d^{2} F}{d x^{2}}+\omega_{2}^{2} F=0
$$

where

$$
R=\frac{\int_{-1 / 2}^{1 / 2}\left(-W_{0}^{2}-2 y W_{0} \frac{d W_{0}}{d y}+2 W_{0} \frac{d W_{1}}{d y}\right) d y}{\int_{-1 / 2}^{1 / 2} \frac{\hat{v}^{2}}{v^{2}(y)} W_{0}^{2} d y} .
$$

The coefficient $R$ encapsulates information about the properties of the microstructure. Knowing the macroscopic field $F(x)$, equation (71) allows evaluating the next term $\omega_{2}^{2}$ of the frequency expansion. For the case of harmonic waves $F(x)$ is given by expression (38); then $\omega_{2}^{2}=4 \pi^{2} R$.

\subsection{Piecewise homogeneous periodic medium}

As an illustrative example, let us consider a piecewise homogeneous structure consisting of alternating layers of two different components $\Gamma^{(1)}$ and $\Gamma^{(2)}$ with a perfect bonding at the interface $\partial \Gamma$ (Fig. 10).

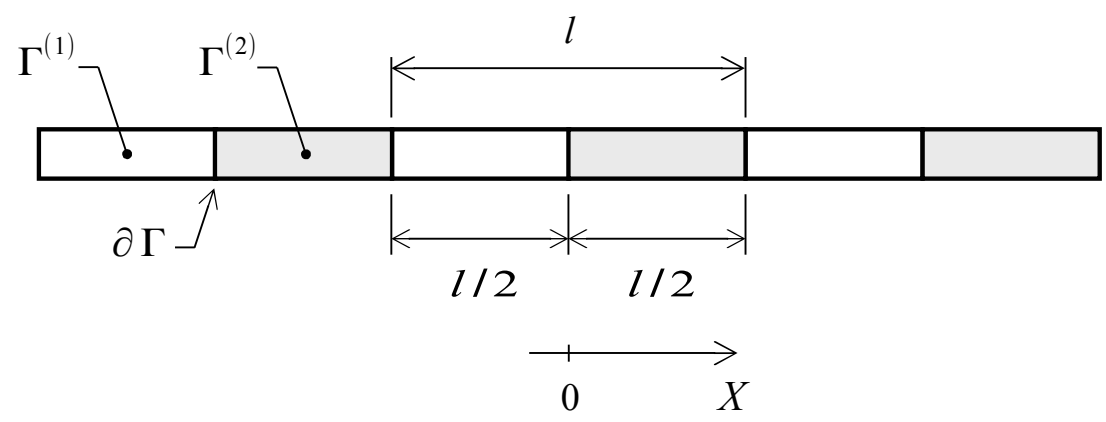

Fig. 10. Piecewise homogeneous medium. 
The exact dispersion relation can be obtained by the Floquet-Bloch approach (see, for example, Andrianov et al. (2008)), which yields

$$
\pm \cos (k)=\cos \left(\frac{\alpha^{(1)} \omega}{2}\right) \cos \left(\frac{\alpha^{(2)} \omega}{2}\right)-\frac{1}{2}\left(\lambda+\frac{1}{\lambda}\right) \sin \left(\frac{\alpha^{(1)} \omega}{2}\right) \sin \left(\frac{\alpha^{(2)} \omega}{2}\right)
$$

with "+" for periodic and with "-" is for anti-periodic modes. Here and in the sequel the upper indexes $(m), m=1,2$, refer to the different components $\Gamma^{(1)}, \Gamma^{(2)}$ of the structure. $\alpha^{(m)}=\hat{v} / v^{(m)} ; v^{(m)}$ is the wave speed in the $m$ th component; $\hat{v}=\left(v^{(1)}+v^{(2)}\right) / 2$. It is easy to show that $\alpha^{(1)}=(1+\lambda) / 2$, $\alpha^{(2)}=(1+1 / \lambda) / 2$, where $\lambda=v^{(2)} / v^{(1)}$. The parameter $\lambda$ characterises the contrast between the properties of the layers.

We now apply the asymptotic procedure described in Section 3.1. The eigenvalue problem of the leading order reads

$$
\begin{gathered}
\frac{d^{2} W_{0}^{(m)}}{d y^{2}}+\left(\alpha^{(m)} \omega_{0}\right)^{2} W_{0}^{(m)}=0 \\
\left.W_{0}^{(1)}\right|_{y=0}=\left.W_{0}^{(2)}\right|_{y=0},\left.\quad \frac{d W_{0}^{(1)}}{d y}\right|_{y=0}=\left.\frac{d W_{0}^{(2)}}{d y}\right|_{y=0}, \\
\left.W_{0}^{(1)}\right|_{y=-\frac{1}{2}}= \pm\left. W_{0}^{(2)}\right|_{y=\frac{1}{2}},\left.\quad \frac{d W_{0}^{(1)}}{d y}\right|_{y=-\frac{1}{2}}= \pm\left.\frac{d W_{0}^{(2)}}{d y}\right|_{y=\frac{1}{2}} .
\end{gathered}
$$

In (75) “+” accounts for periodic and "-" for anti-periodic modes.

Solution of equation (73) is

$$
W_{0}^{(m)}=A_{0}^{(m)} \sin \left(\alpha^{(m)} \omega_{0} y\right)+B_{0}^{(m)} \cos \left(\alpha^{(m)} \omega_{0} y\right) .
$$

Substituting (76) into the boundary conditions (74), (75), we obtain a system of four linear algebraic equations for the unknown amplitudes $A_{0}^{(m)}, B_{0}^{(m)}$. Equating the determinant of the matrix of the coefficients to zero, we derive the equation for $\omega_{0}$ :

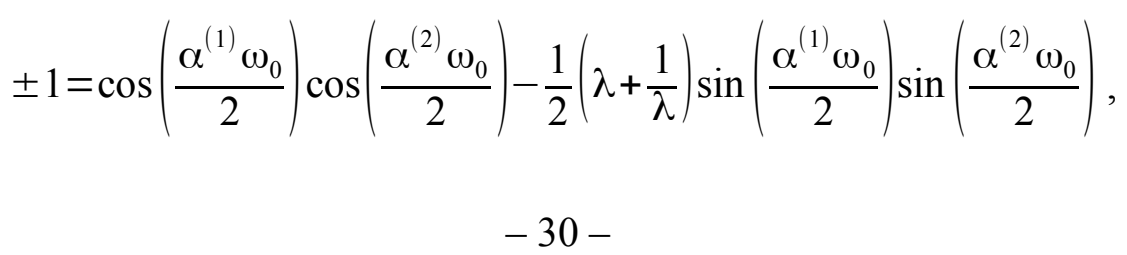


where "+" is for periodic and "_" is for anti-periodic modes.

In the first order approximation, the function $W_{1}^{(m)}$ is obtained as follows

$$
W_{1}^{(m)}=A_{1}^{(m)} \sin \left(\alpha^{(m)} \omega_{0} y\right)+B_{1}^{(m)} \cos \left(\alpha^{(m)} \omega_{0} y\right),
$$

with the coefficients $A_{1}^{(m)}, B_{1}^{(m)}$ being subject to the boundary conditions

$$
\begin{gathered}
\left.w_{1}^{(1)}\right|_{y=0}=\left.w_{1}^{(2)}\right|_{y=0},\left.\quad \frac{d w_{1}^{(1)}}{d y}\right|_{y=0}=\left.\frac{d w_{1}^{(2)}}{d y}\right|_{y=0}, \\
\left.w_{1}^{(1)}\right|_{y=-\frac{1}{2}}= \pm\left. w_{1}^{(2)}\right|_{y=\frac{1}{2}},\left.\quad \frac{d w_{1}^{(1)}}{d y}\right|_{y=-\frac{1}{2}}= \pm\left.\frac{d w_{1}^{(2)}}{d y}\right|_{y=\frac{1}{2}} .
\end{gathered}
$$

In (80), "+" is for periodic and "-" is for anti-periodic modes. We note that $W_{1}^{(m)}$ and $w_{1}^{(m)}$ are related by expression (67).

In order to evaluate integrals in formula (71) and to derive $\omega_{2}^{2}$, we need to determine the amplitudes $A_{0}^{(m)}, B_{0}^{(m)}, A_{1}^{(m)}, B_{1}^{(m)}$. Without loss of generality, let us introduce the local boundary conditions as follows

$$
\begin{gathered}
\left.W_{0}^{(1)}\right|_{y=0}=\left.W_{0}^{(2)}\right|_{y=0}=A, \\
\left.w_{1}^{(1)}\right|_{y=0}=\left.w_{1}^{(2)}\right|_{y=0}=0 ;
\end{gathered}
$$

where $A$ is the amplitude of the wave field. Substituting expression (76) into (74), (75), (81) and expressions (67), (78) into (79), (80), (82), we obtain for periodic modes

$$
A_{0}^{(1)}=A \frac{\cos \left(\frac{\alpha^{(1)} \omega_{0}}{2}\right)-\cos \left(\frac{\alpha^{(2)} \omega_{0}}{2}\right)}{\sin \left(\frac{\alpha^{(1)} \omega_{0}}{2}\right)+\lambda \sin \left(\frac{\alpha^{(2)} \omega_{0}}{2}\right)}, \quad A_{0}^{(2)}=\lambda A_{0}^{(1)}, \quad B_{0}^{(1)}=B_{0}^{(2)}=A
$$




$$
A_{1}^{(1)}=A \frac{\sin \left(\frac{\alpha^{(1)} \omega_{0}}{2}\right) \cos \left(\frac{\alpha^{(2)} \omega_{0}}{2}\right)+\lambda \cos \left(\frac{\alpha^{(1)} \omega_{0}}{2}\right) \sin \left(\frac{\alpha^{(2)} \omega_{0}}{2}\right)}{\left[\sin \left(\frac{\alpha^{(1)} \omega_{0}}{2}\right)+\lambda \sin \left(\frac{\alpha^{(2)} \omega_{0}}{2}\right)\right]^{2}}, \quad A_{1}^{(2)}=\lambda A_{1}^{(1)}, \quad B_{1}^{(1)}=B_{1}^{(2)}=0 ;
$$

and for anti-periodic modes

$$
\begin{aligned}
& A_{0}^{(1)}=A \frac{\cos \left(\frac{\alpha^{(1)} \omega_{0}}{2}\right)+\cos \left(\frac{\alpha^{(2)} \omega_{0}}{2}\right)}{\sin \left(\frac{\alpha^{(1)} \omega_{0}}{2}\right)-\lambda \sin \left(\frac{\alpha^{(2)} \omega_{0}}{2}\right)}, A_{0}^{(2)}=\lambda A_{0}^{(1)}, \quad B_{0}^{(1)}=B_{0}^{(2)}=A ; \\
& A_{1}^{(1)}=-A \frac{\sin \left(\frac{\alpha^{(1)} \omega_{0}}{2}\right) \cos \left(\frac{\alpha^{(2)} \omega_{0}}{2}\right)+\lambda \cos \left(\frac{\alpha^{(1)} \omega_{0}}{2}\right) \sin \left(\frac{\alpha^{(2)} \omega_{0}}{2}\right)}{\left[\sin \left(\frac{\alpha^{(1)} \omega_{0}}{2}\right)-\lambda \sin \left(\frac{\alpha^{(2)} \omega_{0}}{2}\right)\right]^{2}}, A_{1}^{(2)}=\lambda A_{1}^{(1)}, \quad B_{1}^{(1)}=B_{1}^{(2)}=0 .
\end{aligned}
$$

Then, evaluating integrals in (71) and taking into account $\omega_{2}^{2}=4 \pi^{2} R$, we derive

$$
\omega_{2}^{2}= \pm \frac{32 \pi^{2} \lambda^{2} \omega_{0}}{\lambda(1+\lambda)\left(3+\lambda^{2}\right) \cos \left(\frac{\alpha^{(1)} \omega_{0}}{2}\right) \sin \left(\frac{\alpha^{(2)} \omega_{0}}{2}\right)+(1+\lambda)\left(1+3 \lambda^{2}\right) \sin \left(\frac{\alpha^{(1)} \omega_{0}}{2}\right) \cos \left(\frac{\alpha^{(2)} \omega_{0}}{4}\right)}
$$

where "+" is for periodic and "_" is for anti-periodic modes.

For the lowest (acoustic) branch of the dispersion diagram in the periodic case $\omega_{0}=0$ and formula (85) gives $\omega_{2}^{2}=8 \lambda^{2} /\left[(1+\lambda)^{2}\left(1+\lambda^{2}\right)\right]$.

We have evaluated the frequency expansion (31) up to $O\left(\varepsilon^{2}\right)$. Then, a macroscopic model applicable within the entire region $0 \leq k \leq \pi$ can be derived in the same way as it was presented in Section 2.3. Matching together the asymptotic expansions of $\omega^{2}$ at $k \rightarrow 0$ and $k \rightarrow \pi, k=2 \pi \varepsilon$, different macroscopic equations can be obtained for every pass band.

As a numerical example, let us consider the acoustic branch, which describes the lowest part of the spectrum and, therefore, is of primary importance for many engineering applications. For the periodic mode, the macroscopic equation has entirely the same form as (26), where the coefficients $a_{1}, \ldots, a_{4}$ are related to the frequency expansions by formulas (22). The dispersion diagram is 
displayed at Fig. 11. Within the pass band, the developed macroscopic model (26) demonstrates a very good agreement with the exact solution (72) and in the stop band it provides an asymptotic approximation to the attenuation coefficient.
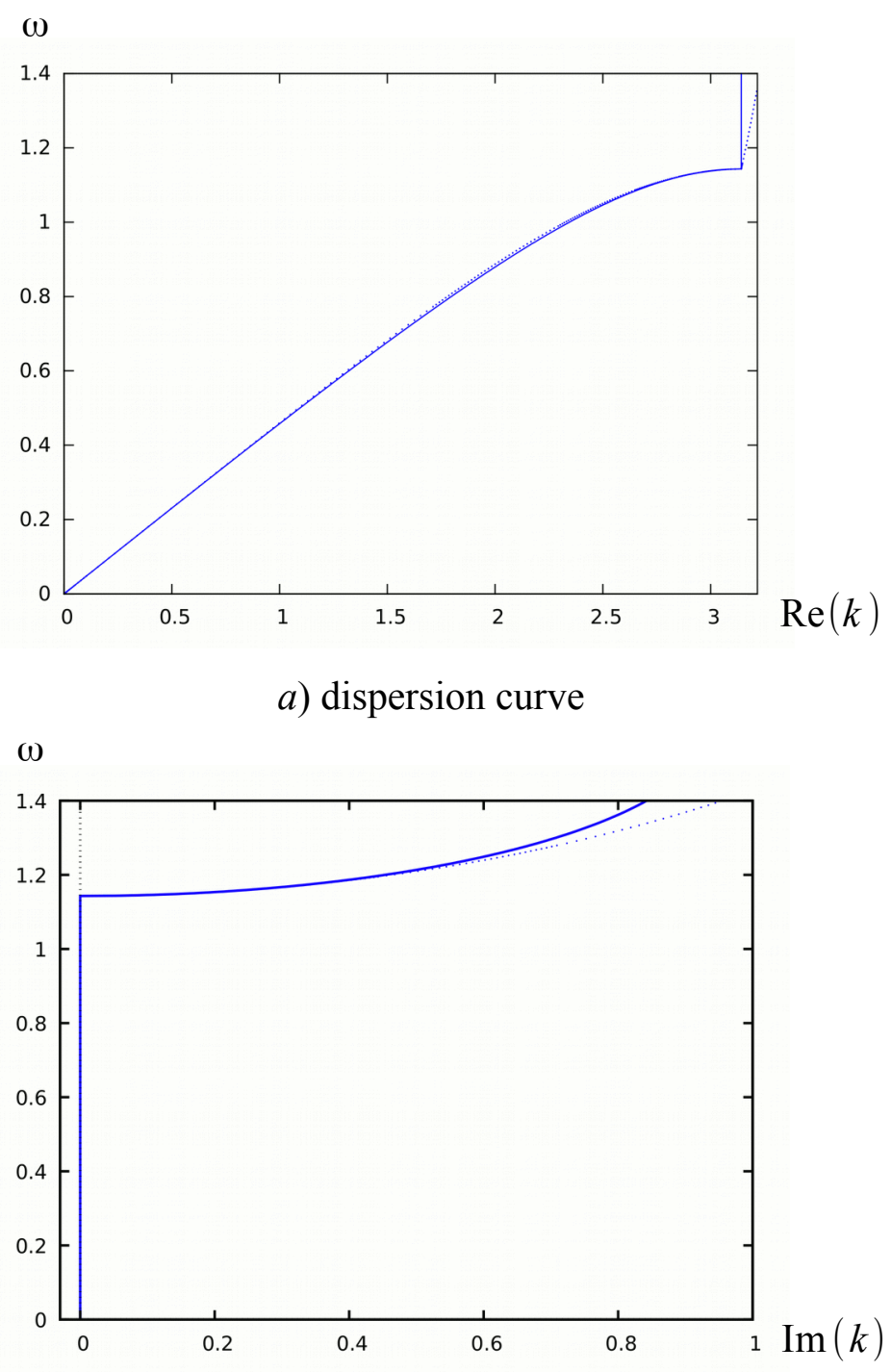

b) attenuation coefficient

Fig. 11. Dispersion diagram of the acoustic periodic mode in the piecewise homogeneous medium; $\lambda=0.2$. Solid curves - exact solution (72), dotted curves - macroscopic model (26).

\subsection{High-contrast periodic media}

A special case of interest is to consider heterogeneous media that include components with high-contrast physical properties. For such kind of structures, the leading order term $\omega_{0}^{2}$ of the 
frequency expansion (31) decreases for all types of the modes. Thus, the dispersion curves "flatten" and shift to the low-frequency domain. The medium can exhibit stop-band and to block wave propagation even at very low frequencies.

Let us consider the piecewise homogeneous structure displayed at Fig. 10, when the wave speeds $v^{(1)}, v^{(2)}$ in the components are strongly different. The asymptotic solution can be easily derived using $\lambda$ as a small parameter. Letting $\lambda \rightarrow 0$, equation (77) gives

$$
\omega_{0}=C \lambda+O\left(\lambda^{2}\right)
$$

where the constant $C$ is evaluated from the equation

$$
\pm 1=\cos \left(\frac{C}{4}\right)-\frac{C}{8} \sin \left(\frac{C}{4}\right)
$$

with "+" for periodic and “-” for anti-periodic modes.

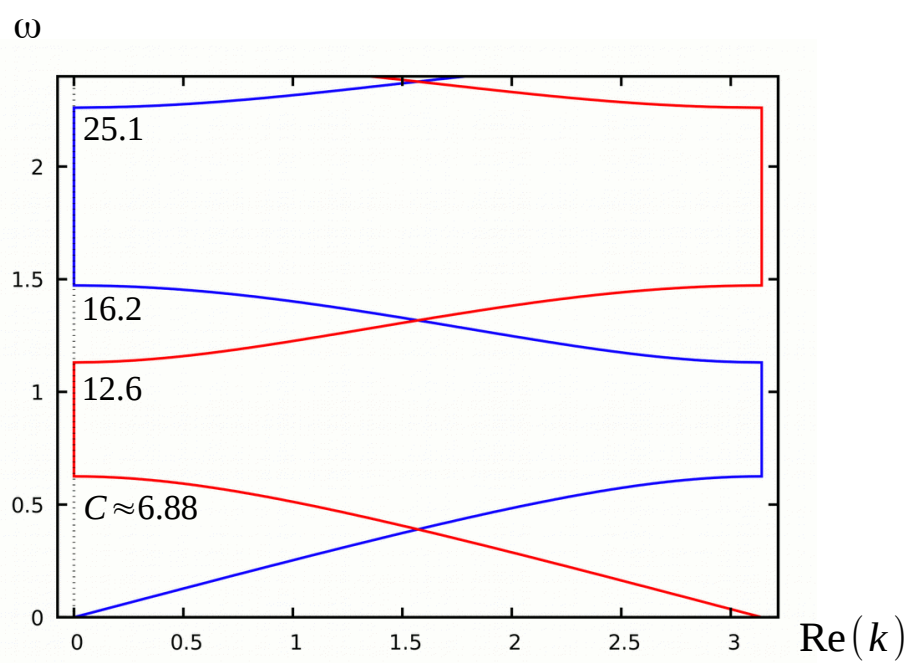

Fig. 12. Dispersion curves for periodic (blue) and anti-periodic (red) modes in the piecewise homogeneous medium; $\lambda=0.1$

We recall that $\omega_{0}$ determines the edges of the phononic bands. In order to illustrate this, the dispersion curves are sketched at Fig. 12 for $\lambda=0.1$. For every curve, we indicate the magnitude of the constant $C$ that determines $\omega_{0}$ in the long-wave limit as $k \rightarrow 0$. The first stop-band threshold is given by the lowest non-zero root of equation (87) at $C \approx 6.88$ and with the 1.h.s. equals minus one. The corresponding value of $\omega_{0}$ is the lowest anti-periodic resonant frequency of the unit cell. The 
numerical results for $\omega_{0}$ are displayed at Fig. $13 a$. We note that $\omega_{0}$ vanishes as $\lambda \rightarrow 0$ and the asymptotic estimation (86) provides a good numerical accuracy for $\lambda<10^{-1}$.

In the example above we have studied a structure consisting of components of the same elastic constants, but with different wave speeds. Now let us turn to the case, when the components have non-equal elastic constants, but possess the same wave speeds. The input model is given by equation (61), where $E(x), \rho(x)=E^{(m)}, \rho^{(m)}$ as $x \in \Gamma^{(m)}$. We assume $v^{(1)}=v^{(2)}$, where $v^{(m)}=E^{(m)} / \rho^{(m)}$, and introduce the contrast parameter $\delta$ as follows $\delta=E^{(2)} / E^{(1)}=\rho^{(2)} / \rho^{(1)}$.

Equation for $\omega_{0}$ takes the form

$$
\pm 1=1-\frac{(1+\delta)^{2}}{4 \delta}\left[1-\cos \left(\omega_{0}\right)\right]
$$

where "+" is for periodic and "-" is for anti-periodic modes. Setting $\delta \rightarrow 0$, an asymptotic estimation of $\omega_{0}$ can be obtained in the high-contrast case. For the first stop-band threshold we get

$$
\omega_{0}=4 \delta^{(1 / 2)}[1+O(\delta)]
$$

The numerical results are presented at Fig. 13b. We can observe that the asymptotic formula (89) shows a good numerical accuracy when $\delta$ is by the order of magnitude about $10^{-1}$ or smaller.

In high-contrast media the energy of deformation is localised within the soft component, while the stiff component undergoes a nearly rigid-body motion. Such pattern of the deformation represents a clear analogue to the propagation of high-frequency long waves in thin-walled elastic waveguides (Craster et al., 2014). The local solution across the unit cell of a heterogeneous medium corresponds to the solution within the transverse cross section of a thin rod, plate or shell.

Let us analyse the displacement field in the unit cell at the long-wave limit for the acoustic anti-periodic mode. This case corresponds to the first stop-band threshold. The solution is given by expression (76). In the case under consideration $E^{(1)} / E^{(2)}=\rho^{(1)} / \rho^{(2)}$ and, therefore $\alpha^{(1)}=\alpha^{(2)}=1$. The coefficients $A_{0}^{(m)}, B_{0}^{(m)}$ read

$$
A_{0}^{(2)}=-A \frac{2 \cos \left(\omega_{0} / 2\right)}{(1-\delta) \sin \left(\omega_{0} / 2\right)}, \quad A_{0}^{(1)}=\delta A_{0}^{(2)}, \quad B_{0}^{(1)}=B_{0}^{(2)}=A
$$




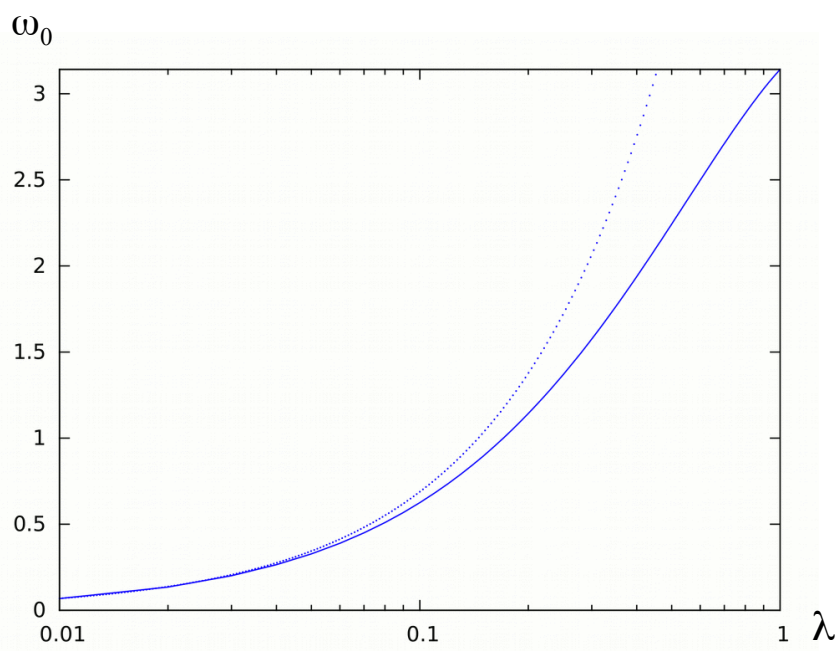

a) high-contrast wave speeds in the components

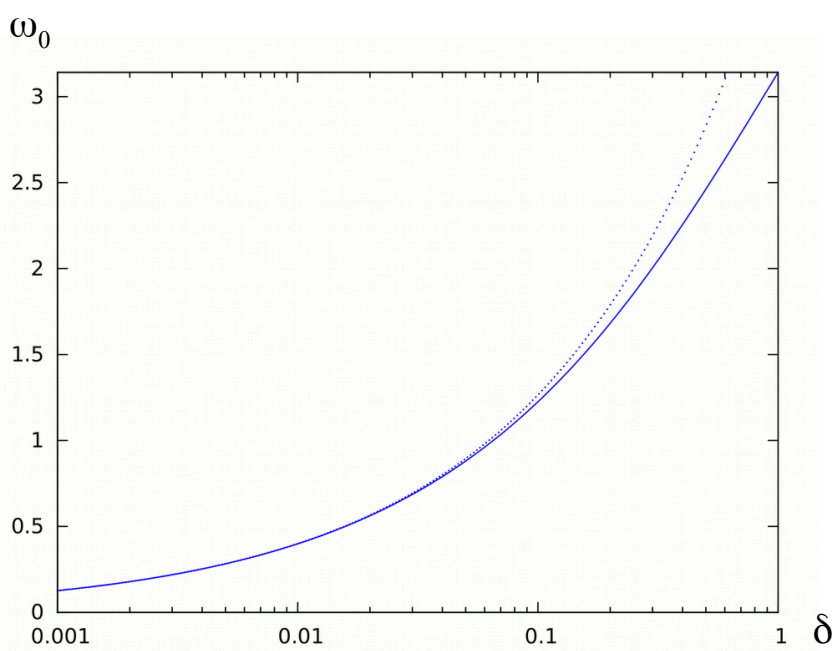

b) high-contrast elastic constants of the components

Fig. 13. The first stop-band threshold of high-contrast piecewise homogeneous media.

Solid curves - exact solution (77), (88), dotted curves - asymptotic results (86), (89).

It should be highlighted that if the elastic constants of the components are allowed to be not equal each other, $E^{(2)} / E^{(1)} \neq 1$, then in the high-contrast case the exact solution (76), (90) for the displacement field can be expressed asymptotically in terms of polynomial functions. Letting $\delta \rightarrow 0$, one obtains

$$
W_{0}^{(1)}=A\left[1-4 y(1+2 y) \delta+O\left(\delta^{2}\right)\right], \quad W_{0}^{(2)}=A\left[1-4 y+\frac{4 y-24 y^{2}+32 y^{3}}{3} \delta+O\left(\delta^{2}\right)\right]
$$

Expansions (91) are purely analogous to the asymptotic models of vibration modes in layered thin- 
walled structures that have been proposed very recently by Kaplunov et el. (2017).

Numerical results are displayed at Fig. 14. We observe that with the decrease of $\delta$ only the soft component $\Gamma^{(2)}$ undergoes essential deformation, whereas the stiff component $\Gamma^{(1)}$ moves like an almost undeformed rigid body. At $\delta \sim 10^{-1}$ the asymptotic and the exact solutions become indistinguishable.

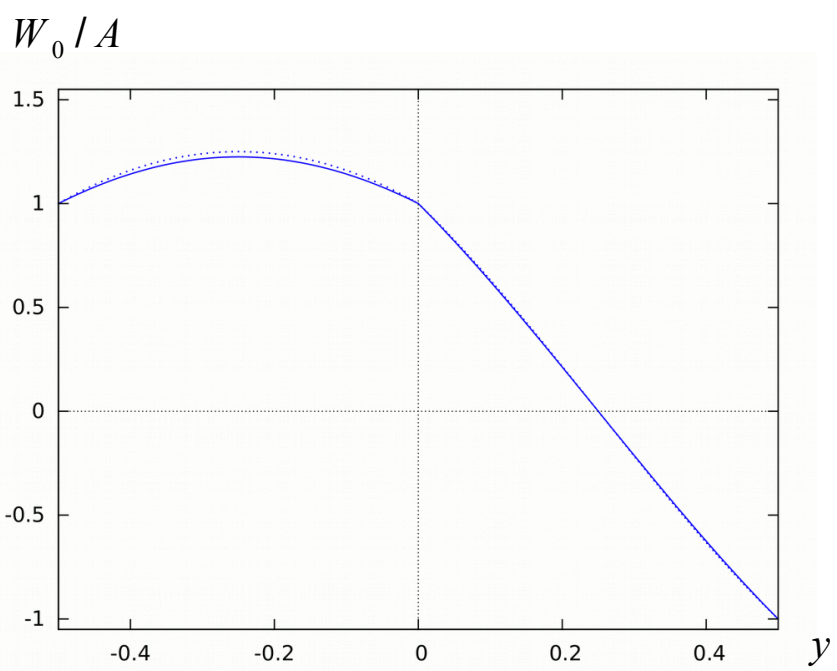

a) $\delta=0.5$

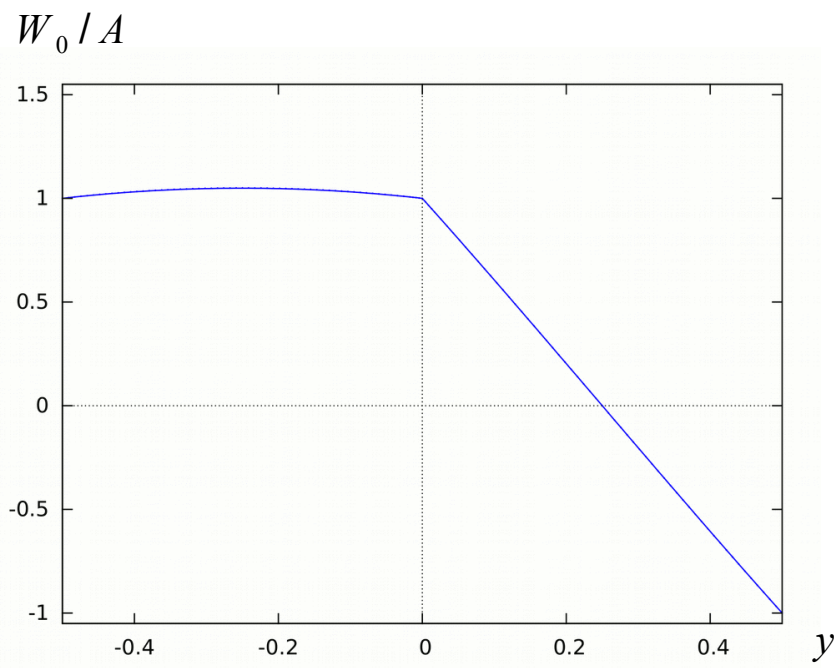

b) $\delta=0.1$

Fig. 14. Displacement field in the unit cell at the long-wave limit at the first stop-band threshold. Solid curves - exact solution (76), (90); dotted curves - asymptotic expressions (91). 


\section{Two-dimensional case}

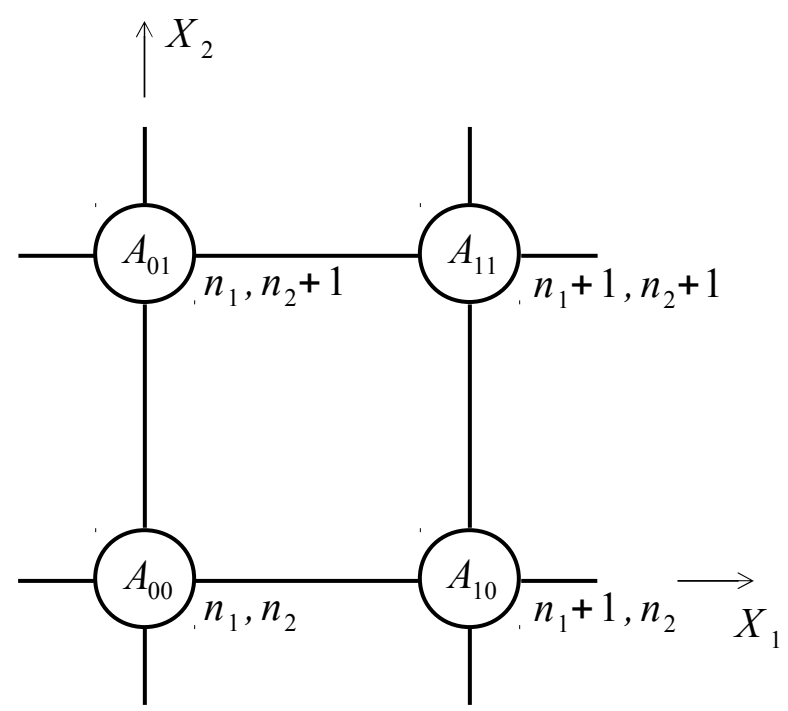

Fig. 15. Square lattice with a four-particles unit cell.

The developed approaches can be further generalised to multi-dimensional problems. As a very brief introduction to the topic, we consider anti-plane waves propagating through a periodic square lattice consisting of identical particles of the mass $m$ connected by springs of the rigidity $c$ (Fig. 15). The displacements $u_{n_{1}, n_{2}}$ of the particles are directed transverse to the plane $X_{1} X_{2}$. Every particle is supposed to interact with its four neighbours. The interaction forces are linear and represent an analogy to shear stresses in a continuous medium.

Let us distinguish a unit cell that includes four particles. We use different notations for the displacements of odd and even particles, i.e.

$$
u_{n_{1}, n_{2}}=\left\{\begin{array}{l}
u_{n_{1}, n_{2}}^{(00)} \text { for } n_{1}, n_{2}= \pm 0, \pm 2, \pm 4, \ldots ; \\
u_{n_{1}, n_{2}}^{(01)} \text { for } n_{1}= \pm 0, \pm 2, \pm 4, \ldots, n_{2}= \pm 1, \pm 3, \pm 5, \ldots \\
u_{n_{1}, n_{2}}^{(10)} \text { for } n_{1}= \pm 1, \pm 3, \pm 5, \ldots, n_{2}= \pm 0, \pm 2, \pm 4, \ldots \\
u_{n_{1}, n_{2}}^{(11)} \text { for } n_{1}, n_{2}= \pm 1, \pm 3, \pm 5, \ldots
\end{array}\right.
$$

The equations of motion for the particles in the unit cell take the form:

$$
m \frac{d^{2} u_{n_{1}, n_{2}}^{(00)}}{d T^{2}}=c\left(u_{n_{1}+1, n_{2}}^{(10)}+u_{n_{1}-1, n_{2}}^{(10)}+u_{n_{1}, n_{2}+1}^{(01)}+u_{n_{1}, n_{2}-1}^{(01)}-4 u_{n_{1}, n_{2}}^{(00)}\right)
$$




$$
\begin{gathered}
m \frac{d^{2} u_{n_{1}, n_{2}+1}^{(01)}}{d T^{2}}=c\left(u_{n_{1}+1, n_{2}+1}^{(11)}+u_{n_{1}-1, n_{2}+1}^{(11)}+u_{n_{1}, n_{2}+2}^{(00)}+u_{n_{1}, n_{2}}^{(00)}-4 u_{n_{1}, n_{2}+1}^{(01)}\right), \\
m \frac{d^{2} u_{n_{1}+1, n_{2}}^{(10)}}{d T^{2}}=c\left(u_{n_{1}+2, n_{2}}^{(00)}+u_{n_{1}, n_{2}}^{(00)}+u_{n_{1}+1, n_{2}+1}^{(11)}+u_{n_{1}+1, n_{2}-1}^{(11)}-4 u_{n_{1}+1, n_{2}}^{(10)}\right), \\
m \frac{d^{2} u_{n_{1}+1, n_{2}+1}^{(11)}}{d T^{2}}=c\left(u_{n_{1}+2, n_{2}+1}^{(01)}+u_{n_{1}, n_{2}+1}^{(01)}+u_{n_{1}+1, n_{2}+2}^{(10)}+u_{n_{1}+1, n_{2}}^{(10)}-4 u_{n_{1}+1, n_{2}+1}^{(11)}\right) .
\end{gathered}
$$

Let us suppose that the particles in the unit cell can vibrate with different amplitudes $A_{00}$, $A_{01}, A_{10}, A_{11}$, i.e.

$$
u_{n_{1}, n_{2}}^{\left(j_{1} j_{2}\right)}=A_{j_{1} j_{2}} \exp (i \Omega T) \exp \left[-i\left(k_{1} n_{1}+k_{2} n_{2}\right)\right], \quad j_{1} j_{2}=0,1
$$

where $k_{1}, k_{2}$ are projections of the non-dimensional wave vector $\mathbf{k}$ onto the coordinate axes.

Substituting (93) into (92), we come to a system of equations for the unknown amplitudes. In the matrix form it reads

$$
\left[\mathbf{M}(\mathbf{k})+\omega^{2} \mathbf{I}_{4}\right] \cdot \mathbf{A}=0,
$$

where $\omega=\Omega(m / c)^{1 / 2}, \mathbf{A}=\left[A_{00}, A_{01}, A_{10}, A_{11}\right]^{T} ; \mathbf{I}_{4}$ is the identity matrix of the 4th order; $\mathbf{M}$ is the symmetric matrix as follows

$$
\mathbf{M}=\left[\begin{array}{cccc}
-4 & 2 \cos \left(k_{2}\right) & 2 \cos \left(k_{1}\right) & 0 \\
\cdots & -4 & 0 & 2 \cos \left(k_{1}\right) \\
\ldots & \ldots & -4 & 2 \cos \left(k_{2}\right) \\
\cdots & \ldots & \ldots & -4
\end{array}\right]
$$

Equation (94) determines the eigenvalue problem that allows to evaluate the dispersion relation. Depending on the ratio between the amplitudes of the particles, four different types of waves can be distinguished.

1. $A_{00}=A_{01}=A_{10}=A_{11}$. In this case the particles in the unit cell move in-phase. In the longwave limit as $k_{1}, k_{2} \rightarrow 0$ one obtains a solution, which is periodic across the unit cell in the both directions $X_{1}$ and $X_{2}$. We refer this type of wave as a periodic mode and will denote it by the abbreviation P1P2. This is a conventional acoustic mode that appears in the classical theory. The 
dispersion relation is as follows

$$
\omega^{2}=4\left[\sin ^{2}\left(\frac{k_{1}}{2}\right)+\sin ^{2}\left(\frac{k_{2}}{2}\right)\right] .
$$

2. $A_{00}=A_{01}=-A_{10}=-A_{11}$. The neighbouring particles move out-of-phase in the direction $X_{1}$ and in-phase in the direction $X_{2}$. In the long-wave limit the solution is locally anti-periodic by $X_{1}$ and periodic by $X_{2}$. This mode is denoted as A1P2. The dispersion relation reads

$$
\omega^{2}=4\left[\cos ^{2}\left(\frac{k_{1}}{2}\right)+\sin ^{2}\left(\frac{k_{2}}{2}\right)\right] .
$$

3. $A_{00}=-A_{01}=A_{10}=-A_{11}$. The wave is periodic by $X_{1}$ and anti-periodic by $X_{2}$ (P1A2 mode). The solution is identical to the case 2 within the interchanging $k_{1} \Leftrightarrow k_{2}$ :

$$
\omega^{2}=4\left[\sin ^{2}\left(\frac{k_{1}}{2}\right)+\cos ^{2}\left(\frac{k_{2}}{2}\right)\right] .
$$

4. $A_{00}=-A_{01}=-A_{10}=A_{11}$. In this case the solution is anti-periodic in the both directions $X_{1}$ and $X_{2}$ (A1A2 mode). The dispersion relation is

$$
\omega^{2}=4\left[\cos ^{2}\left(\frac{k_{1}}{2}\right)+\cos ^{2}\left(\frac{k_{2}}{2}\right)\right] .
$$

The dispersion curves are presented at Fig. 16. The right part of the diagram displays the solution for the orthogonal $X_{1}$ direction of wave propagation $\left(k_{1}=k, k_{2}=0\right)$ and the left part corresponds to the diagonal direction $\left(k_{1}=k_{2}=k / \sqrt{2}\right)$. 


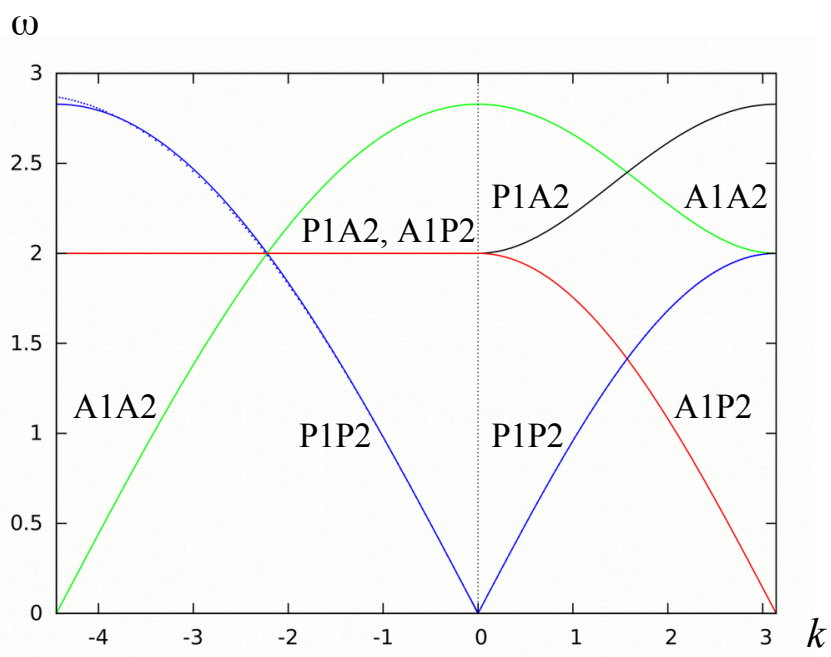

Fig. 16. Dispersion curves of the square lattice.

Blue - P1P2 mode; red - A1P2 mode; black - P1A2 mode; green - A1A2 mode.

Solid curves - exact solutions (95)-(98), dotted curves - macroscopic model (117).

In the long-wave limit the frequency of the periodic mode P1P2 vanishes, $\omega \rightarrow 0$ as $k \rightarrow 0$, so no vibrations occur. Alternatively, all the anti-periodic modes turn to standing waves with zero group velocities and non-zero frequencies. As an example, let us analyse the waves propagating in the direction $X_{1}$ (the right part of the diagram at Fig. 16). The modes P1P2 and A1P2 turn to each other when the wave number $k$ is shifted to a half of its period: $k \Leftrightarrow \pi-k$. Meanwhile, the modes P1A2 and A1A2 describe a particularly different type of motion, when the wave is anti-periodic in the direction transverse to the direction of propagation. Such types of waves arise specifically in multi-dimensional structures and they cannot be described by one-dimensional models.

In order to develop long-wave continuous approximations, let us introduce the effective wave field $\widetilde{u}_{n_{1}, n_{2}}$ describing propagation of the envelope wave. We denote

$$
\widetilde{u}_{n_{1}, n_{2}}=\frac{u_{n_{1}, n_{2}}^{(00)}+j_{1} u_{n_{1}, n_{2}+1}^{(01)}+j_{2} u_{n_{1}+1, n_{2}}^{(10)}+j_{3} u_{n_{1}+1, n_{2}+1}^{(11)}}{4}
$$

with $j_{1}=j_{2}=j_{3}=1$ for P1P2 mode; $j_{1}=1, j_{2}=j_{3}=-1$ for A1P2 mode; $j_{1}=j_{3}=-1, j_{2}=1$ for P1A2 mode; $j_{1}=j_{2}=-1, j_{3}=1$ for A1A2 mode.

With respect to the new field function $\tilde{u}_{n_{1}, n_{2}}$, equations (92) become uncoupled and read

$$
m \frac{d^{2} \widetilde{u}_{n_{1}, n_{2}}}{d T^{2}}=c\left[j_{1}\left(\widetilde{u}_{n_{1}, n_{2}+1}+\widetilde{u}_{n_{1}, n_{2}-1}\right)+j_{2}\left(\widetilde{u}_{n_{1}+1, n_{2}}+\widetilde{u}_{n_{1}-1, n_{2}}\right)-4 \widetilde{u}_{n_{1}, n_{2}}\right] .
$$


A spatially continuous function $u\left(X_{1}, X_{2}, T\right)$ describing the displacement of the lattice is introduced as follows:

$$
u\left(X_{1}, X_{2}, T\right)=\widetilde{u}_{n_{1}, n_{2}}(T) \text { at } X_{\alpha}=n_{\alpha} l, \alpha=1,2
$$

where $l$ is the distance between the particles. Using a Taylor series expansion, let us represent

$$
\widetilde{u}_{n_{1} \pm 1, n_{2} \pm 1}(T)=u\left(x_{1} \pm \varepsilon, \bar{x}_{2} \pm \varepsilon, T\right)=\sum_{p=0}^{\infty} \frac{D^{p}\left[u\left(x_{1}, x_{2}, T\right)\right]}{p !}
$$

where $D=\varepsilon\left( \pm \partial / \partial x_{1} \pm \partial / \partial x_{2}\right) ; x_{\alpha}=X_{\alpha} / L ; \varepsilon=l / L \ll 1 ; \quad l$ is the distance between the particles; $L$ is the wavelength.

Substituting (100), (101) into (99) gives higher-order macroscopic equations that describe the dynamics of the lattice in the long-wave case as $\varepsilon \rightarrow 0$. Within the error $O\left(\varepsilon^{6}\right)$ we obtain

for P1P2 mode

$$
\varepsilon^{2}\left(\frac{\partial^{2} u}{\partial x_{1}^{2}}+\frac{\partial^{2} u}{\partial x_{2}^{2}}\right)+\frac{\varepsilon^{4}}{12}\left(\frac{\partial^{4} u}{\partial x_{1}^{4}}+\frac{\partial^{4} u}{\partial x_{2}^{4}}\right)-\frac{\partial^{2} u}{\partial t^{2}}=0
$$

for A1P2 mode

$$
4 u+\varepsilon^{2}\left(\frac{\partial^{2} u}{\partial x_{1}^{2}}-\frac{\partial^{2} u}{\partial x_{2}^{2}}\right)+\frac{\varepsilon^{4}}{12}\left(\frac{\partial^{4} u}{\partial x_{1}^{4}}-\frac{\partial^{4} u}{\partial x_{2}^{4}}\right)+\frac{\partial^{2} u}{\partial t^{2}}=0 \text {, }
$$

for P1A2 mode

$$
4 u+\varepsilon^{2}\left(\frac{\partial^{2} u}{\partial \bar{x}_{2}^{2}}-\frac{\partial^{2} u}{\partial \bar{x}_{1}^{2}}\right)+\frac{\varepsilon^{4}}{12}\left(\frac{\partial^{4} u}{\partial \bar{x}_{2}^{4}}-\frac{\partial^{4} u}{\partial \bar{x}_{1}^{4}}\right)+\frac{\partial^{2} u}{\partial \bar{t}^{2}}=0 \text {, }
$$

for A1A2 mode

$$
8 u+\varepsilon^{2}\left(\frac{\partial^{2} u}{\partial x_{1}^{2}}+\frac{\partial^{2} u}{\partial x_{2}^{2}}\right)+\frac{\varepsilon^{4}}{12}\left(\frac{\partial^{4} u}{\partial x_{1}^{4}}+\frac{\partial^{4} u}{\partial x_{2}^{4}}\right)+\frac{\partial^{2} u}{\partial t^{2}}=0 \text {. }
$$

where $t=T(\mathrm{c} / \mathrm{m})^{1 / 2}$.

Considering propagation of a harmonic wave

$$
u=A \exp (i \omega t) \exp \left[-i\left(k_{1} y_{1}+k_{2} y_{2}\right)\right]
$$

where $y_{\alpha}=X_{\alpha} / l$, equations (102)-(105) give the asymptotic dispersion relations: 
for P1P2 mode

$$
\omega^{2}=\left(k_{1}^{2}+k_{2}^{2}\right)-\frac{1}{12}\left(k_{1}^{4}+k_{2}^{4}\right)
$$

for A1P2 mode

$\omega^{2}=4-k_{1}^{2}+k_{2}^{2}+\frac{1}{12}\left(k_{1}^{4}-k_{2}^{4}\right)$,

for P1A2 mode

$\omega^{2}=4+k_{1}^{2}-k_{2}^{2}+\frac{1}{12}\left(k_{2}^{4}-k_{1}^{4}\right)$,

for A1A2 mode

$$
\omega^{2}=8-k_{1}^{2}-k_{2}^{2}+\frac{1}{12}\left(k_{1}^{4}+k_{2}^{4}\right) \text {. }
$$

Here the components of the non-dimensional $\mathbf{k}$ and the dimensional $\mathbf{K}$ wave vectors are related as follows: $k_{\alpha}=K_{\alpha} l$, where $K=|\mathbf{K}|=2 \pi / L$. Expressions (107)-(110) coincide with the Taylor series expansions of the exact dispersion relations (95)-(98) as $k_{1}, k_{2} \rightarrow 0$ within the error $O\left(k_{1}^{6}+k_{2}^{6}\right)$.

In order to analyse the dynamic anisotropy of the lattice, let us represent

$$
k_{1}=k \cos (\varphi), \quad k_{2}=k \sin (\varphi)
$$

where $\varphi$ is the angel between the axis $x_{1}$ and the wave vector $\mathbf{k}$. Substituting (111) into (107)-(110) we can easily see that for the modes P1P2 and A1A2 the solution is invariant of $\varphi$ up to $O\left(k_{1}^{2}+k_{2}^{2}\right)$. In the long-wave limit the lattice exhibits the isotropic response. However, when the wave length decreases and approaches the dimension of the microstructure, the dynamic properties of the lattice become essentially anisotropic. Meanwhile, the modes A1P2 and P1A2 are anisotropic even in the long-wave limit.

Expansions (107)-(110) may be used to derive macroscopic models applicable in the entire frequency range and capturing information about the propagation of different modes. As an illustrative example, let us consider an approximation for the conventional P1P2 mode. When one or both of the components $k_{1}, k_{2}$ of the wave vector tends to zero, formulas (107)-(110) give

$$
\begin{gathered}
\omega^{2} \sim k_{1}^{2}+k_{2}^{2} \quad \text { as } k_{1}, k_{2} \rightarrow 0 ; \\
\omega^{2} \sim 4-\left(\pi-k_{1}\right)^{2}+k_{2}^{2} \text { as } k_{1} \rightarrow \pi, k_{2} \rightarrow 0 ; \\
\omega^{2}=4+k_{1}^{2}-\left(\pi-k_{2}\right)^{2} \text { as } k_{1} \rightarrow 0, k_{2} \rightarrow \pi .
\end{gathered}
$$

Matching asymptotic expressions (112)-(114) by two-point Padé approximant in two variables $k_{1}, k_{2}$, we obtain 


$$
\omega^{2}=\frac{k_{1}^{2}+k_{2}^{2}+b_{1}\left(k_{1}^{4}+k_{2}^{4}\right)+b_{2}\left(k_{1}^{6}+k_{2}^{6}\right)}{1+b_{3}\left(k_{1}^{2}+k_{2}^{2}\right)+b_{4}\left(k_{1}^{4}+k_{2}^{4}\right)},
$$

where $b_{1}=\left(192-8 \pi^{2}-5 \pi^{4}\right) /\left(5 \pi^{6}\right), b_{2}=8 /\left(5 \pi^{6}\right), b_{3}=\left(5 \pi^{2}-48\right) /\left(20 \pi^{2}\right)$, $b_{4}=\left(192+28 \pi^{2}-5 \pi^{4}\right) /\left(20 \pi^{6}\right)$.

Formula (115) can be equivalently represented as follows

$$
\omega^{2}-k_{1}^{2}-k_{2}^{2}-b_{1}\left(k_{1}^{4}+k_{2}^{4}\right)-b_{2}\left(k_{1}^{6}+k_{2}^{6}\right)+b_{3} \omega^{2}\left(k_{1}^{2}+k_{2}^{2}\right)+b_{4} \omega^{2}\left(k_{1}^{4}+k_{2}^{4}\right)=0 \text {. }
$$

Then, making use of (25), we derive the higher-order macroscopic model in the following form

$$
\begin{aligned}
& \frac{\partial^{2} u}{\partial y_{1}^{2}}+\frac{\partial^{2} u}{\partial y_{2}^{2}}-\frac{\partial^{2} u}{\partial t^{2}}-b_{1}\left(\frac{\partial^{4} u}{\partial y_{1}^{4}}+\frac{\partial^{4} u}{\partial y_{2}^{4}}\right)+b_{2}\left(\frac{\partial^{6} u}{\partial y_{1}^{6}}+\frac{\partial^{6} u}{\partial y_{2}^{6}}\right)+ \\
& b_{3}\left(\frac{\partial^{4} u}{\partial y_{1}^{2} \partial t^{2}}+\frac{\partial^{4} u}{\partial y_{2}^{2} \partial t^{2}}\right)-b_{4}\left(\frac{\partial^{6} u}{\partial y_{1}^{4} \partial t^{2}}+\frac{\partial^{6} u}{\partial y_{2}^{4} \partial t^{2}}\right)=0
\end{aligned}
$$

Despite equation (117) does not match the asymptotic limit $k_{1}, k_{2} \rightarrow \pi$, it is able to describe the propagation of P1P2 mode in a wide range of frequencies. The numerical results for the dispersion curves are shown at Fig. 16 by the dot lines. In the right part of the diagram the derived approximation is visually indistinguishable from the exact solution. In the left part the accuracy is very good except the limit $k_{1}, k_{2} \rightarrow \pi$.

Equation (117) may be utilised for solving macroscopic problems subject to appropriate boundary and initial conditions. Using the proposed approach, generalised macroscopic models capturing other types of modes may be further developed.

\section{Conclusions}

A new approach to predict the dynamic behaviour of periodically heterogeneous elastic media is proposed. In order to clarify the basics of the methodology, we start with a monatomic lattice model and then extend the analysis to one-dimensional continuous waveguides. A two-scale perturbation procedure allows us to derive macroscopic equations of motion valid in the vicinity of the resonant frequencies of the unit cells. Then the obtained asymptotic solutions are matched using the method of two-point Padé approximants. As a result, we derive new higher-order dynamic 
models capable to describe the dynamic response of the medium both in the low and in the high frequency limits. The developed models encapsulate information about the microstructure and, in contrary to many phenomenological theories of gradient elasticity, all the effective coefficients can be determined on a rigorous theoretical basis in terms of microscopic properties of the medium.

The obtained macroscopic equations capture the dispersive properties of the medium, which is justified by comparisons with Floquet-Bloch theory. Moreover, they can be applied to solving boundary value problems. As an illustrative example, we consider transient waves in a semi-infinite lattice excited by pulse and harmonic loads. An important feature of the pulse load problem is that, due to a spatial redistribution of energy, the internal forces arising in a heterogeneous medium can be higher than the magnitude of the initial excitation. This effect may be crucial for the dynamic failure of structures. It should be noted that the developed analytical models demonstrate excellent agreement with the direct numerical solutions.

In high-contrast materials that consist of components with strongly different mechanical properties (e.g., elastic moduli), the dispersion curve of the acoustic mode shifts to the lowfrequency domain. The band gaps widen and the wave propagation can be blocked even at considerably low frequencies. In such a case the energy of deformation is localised within the soft component, while the stiff component undergoes a nearly rigid-body motion. This reveals a fundamental analogy to the wave propagation in thin-walled structures: the local solution on the unit cell may correspond to the solution within the transverse cross section of a thin rod, plate, or shell (see Craster et al., 2014; Kaplunov, 2017).

The developed theory can be further generalised to multi-dimensional problems. As an illustrative example, propagation of anti-plane waves through a square lattice of identical particles is considered. An important feature of the two-dimensional case is that combining different cell resonances in the directions of the translational symmetry makes it possible to detect additional wave modes, which cannot be predicted within the classical continuous framework. Probably, some of these modes may be interpreted as theoretical counterparts of non-classical waves that appear in phenomenological approaches, such as micro-rotational waves in Cosserat continuum and so called "slow" or "second" wave in Biot's theory.

\section{Acknowledgement}

The authors are grateful to the anonymous reviewer, whose valuable comments and suggestions helped to improve the paper. This work has received funding from the European Union's Horizon 2020 research and innovation programme under Marie Sklodowska-Curie grant 
agreement no. 655177.

\section{References}

Aero E., Kuvshinskii E., 1961. Fundamental equations of the theory of elastic media with rotationally interacting particles. Soviet Phys. - Solid State 2, 1272-1281.

Andrianov I.V., Awrejcewicz J., Weichert D., 2010. Improved continuous models for discrete media. Math. Prob. Eng. 2010, 986242.

Andrianov I.V., Bolshakov V.I., Danishevskyy V.V., Weichert D., 2008. Higher-order asymptotic homogenization and wave propagation in periodic composite materials. Proc. R. Soc. Lond. A 464, 1181-1201.

Andrianov I.V., Danishevskyy V.V., Kalamkarov A.L., 2013. Vibration localization in onedimensional linear and nonlinear lattices: discrete and continuum models. Nonlinear Dynamics $72,37-48$.

Andrianov I.V., Danishevskyy V.V., Kushnierov Ie.A., 2014. Spatial localization of linear elastic waves in composite materials with defects. ZAMM 94, 1001-1010.

Andrianov I.V., Starushenko G.A., Weichert D., 2012. Numerical investigation of 1D continuum dynamical models of discrete chain. Z. Angew. Math. Mech. 92, 945-954.

Antonakakis T., Craster R.V., Guenneau S., 2014. Homogenisation for elastic photonic crystals and dynamic anisotropy. J. Mech. Phys. Solids. 71, 84-96.

Askes H., Aifantis E.C., 2011. Gradient elasticity in statics and dynamics: An overview of formulations, length scale identification procedures, finite element implementations and new results. Int. J. Solids Struct. 48, 1962-1990.

Askes H., Metrikine A.V., Pichugin A.V., Bennett T., 2008. Four simplified gradient elasticity models for the simulation of dispersive wave propagation. Philos. Mag. 88, 1-11.

Auriault J.-L., Boutin C., 2012. Long wavelength inner-resonance cut-off frequencies in elastic composite materials. Int. J. Solids Struct. 49, 3269-3281.

Ayzenberg-Stepanenko M. V., Slepyan L.I., 2008. Resonant-frequency primitive waveforms and star waves in lattices. J. Sound Vib. 313, 812-821.

Baker G.A., Graves-Morris P., 1996. Padé Approximants, 2nd ed. Cambridge University Press, Cambridge.

Bakhvalov N., Panasenko G., 1989. Homogenization: Averaging Processes in Periodic Media. Kluwer, Dordrecht.

Berezovski A., Engelbrecht J., Berezovski M., 2011. Waves in microstructured solids: a unified 
viewpoint of modeling. Acta Mech. 220, 349-363.

Bensoussan A., Lions J.-L., Papanicolaou G., 1978. Asymptotic Analysis for Periodic Structures. North-Holland, Amsterdam.

Bloch F., 1928. Über die Quantenmechanik der Elektronen in Kristallgittern. Z. Physik 52, 555600.

Boutin C., Auriault J.L., 1993. Rayleigh scattering in elastic composite materials. Int. J. Eng. Sci. $31,1669-1689$.

Boutin C., Soubestre J., Dietz M.S., Taylor C., 2013. Experimental evidence of the high-gradient behaviour of fiber reinforced materials. Eur. J. Mech. A-Solid. 42, 280-298.

Brillouin L., 2003. Wave Propagation in Periodic Structures: Electric Filters and Crystal Lattices, 2nd edn. Dover, Mineola, New York.

Brun M., Guenneau S., Movchan A.B., 2009. Achieving control of in-plane elastic waves. Appl. Phys. Lett. 94, 061903.

Carta G., Jones I.S., Movchan N.V., Movchan A.B., Nieves M.J., 2017. "Deflecting elastic prism” and unidirectional localisation for waves in chiral elastic systems. Scientific Reports 7, 26.

Cauchy A.L., 1851. Note sur l'équilibré et les mouvements vibratoires des corps solides. C. R. Acad. Paris. 32, 323-326.

Chesnais C., Boutin C., Hans S., 2012. Effects of the local resonance on the wave propagation in periodic frame structures: generalized Newtonian mechanics. J. Ac. Soc. Am. 132, 2873-2886.

Colquitt D.J., Jones I.S., Movchan N.V., Movchan A.B., 2011. Dispersion and localization of elastic waves in materials with microstructure. Proc. R. Soc. A 467, 2874-2895.

Colquitt D.J., Jones I.S., Movchan N.V., Movchan A.B., McPhedran R.C., 2012. Dynamic anisotropy and localization in elastic lattice systems. Waves Random Complex 22, 143-159.

Colquitt D.J., Jones I.S., Movchan N.V., Movchan A.B., Brun M., McPhedran R.C., 2013 Making waves round a structured cloak: Lattices, negative refraction and fringes. Proc. R. Soc. A 469, 20130218.

Colquitt D.J., Craster R.V., Makwana M., 2015. High frequency homogenisation for elastic lattices. Q. J. Mech. Appl. Math. 68, 203-230.

Cosserat E., Cosserat F., 1909. Théorie des corps déformables. A. Hermann et Fils, Paris.

Craster R.V., Joseph L.M., Kaplunov J., 2014. Long-wave asymptotic theories: The connection between functionally graded waveguides and periodic media. Wave Motion 51, 581-588.

Craster R.V., Kaplunov J., Nolde E., Guenneau S., 2012. Bloch dispersion and high frequency homogenization for separable doubly-periodic structures. Wave Motion 49, 333-346. 
Craster R.V., Kaplunov J., Pichugin A.V., 2010a. High frequency homogenization for periodic media. Proc. R. Soc. Lond. A 466, 2341-2362.

Craster R.V., Kaplunov J., Postnova J., 2010b. High-frequency asymptotics, homogenisation and localisation for lattices. Q. J. Mech. Appl. Math. 63, 497-519.

Daya E.M., Potier-Ferry M., 2001. Vibrations of long repetitive structures by a double scale asymptotic method, Struct. Eng. Mech. 12, 215-230.

Eringen A., 1983. On differential equations of nonlocal elasticity and solutions of screw dislocation and surface waves. J. Appl. Phys. 54, 4703-4710.

Filimonov A.M., Kurchanov P.F., Myshkis A.D., 1991. Some unexpected results in the classical problem of vibrations of the string with $n$ beads when $n$ is large. C.R. Acad. Sci. Paris 313, Ser. $1,961-965$.

Fish J, Chen W., 2001. Higher-order homogenization of initial/boundary-value problem. J. Eng. Mech. 127, 1223-1230.

Floquet G., 1883. Sur les équations différentielles linéaires à coefficients périodiques. Ann. École Norm. Sup. 12, 47-88.

Gibson L.J., Ashby M.F., Harley B.A., 2010. Cellular Materials in Nature and Medicine. Cambridge University Press, Cambridge.

Grbic A., Eleftheriades G.V., 2004. Overcoming the diffraction limit with a planar left-handed transmission-line lens. Phys. Rev. Lett. 92, 117403.

Herrmann G., Achenbach J.D., 1968. Application of theories of generalized Cosserat continua to the dynamics of composite materials. In: E. Kröner (Ed.) Mechanics of Generalized Continua. Berlin, Heidelberg, New York, Springer, 69-79.

Hussein M.I., Leamy M.J., Ruzzene M., 2014. Dynamics of phononic materials and structures: historical origins, recent progress, and future outlook. Appl. Mech. Rev. 66, 040802.

Kaplunov J.D., Nolde E.V., Rogerson G.A., 2000. A low-frequency model for dynamic motion in pre-stressed incompressible elastic structures. Proc. R. Soc. A 456, 2589-2610.

Kaplunov J.D., Pichugin A.V., 2009. On rational boundary conditions for higher-order long-wave models. IUTAM Symposium on Scaling in Solid Mechanics. IUTAM Bookseries 10, 81-90.

Kaplunov J.D., Prikazchikov D.A., Sergushova O., 2017. Lowest vibration modes of strongly inhomogeneous elastic structures. In: Mechanics for Materials and Technologies (eds. Altenbach H., Goldstein R.V., Murashkin E.), Springer.

Kittel C., 1996. Introduction to Solid State Physics, 7nd ed. Wiley, New York.

Krein M.G., Liubarskii G.Ia., 1961. On the theory of transmission bands of periodic waveguides. J. Appl. Math. Mech. 25(1), 29-48. 
Kronig R.L., Penney W.G., 1931. Quantum mechanics in crystals lattices. Proc. R. Soc. Lond A 130, 499-531.

Kröner E., 1963. On the physical reality of torque stresses in continuum mechanics. Int. J. Eng. Sci. $1,261-278$.

Kunin I., 1966. Theory of elasticity with spatial dispersion. One-dimensional complex structure. J. Appl. Math. Mech. 30, 1025-1034.

Kushwaha M.S., Halevi P., Martinez G., Dobrzynski L., Djafari-Rouhani B., 1994. Theory of acoustic band structure of periodic elastic composites. Phys. Rev. B 49, 2313-2322.

Le Roux J., 1911. Étude géométrique de la torsion et de la flexion dans la déformation. Ann. Scient. de l'École Normale Sup, 3e série, tome 28, 523-579.

Levin V., 1971. The relation between mathematical expectations of stress and strain tensors in elastic microheterogeneous media. J. Appl. Math. Mech. 35, 694-701.

Liang B., Yuan B., Cheng J.C., 2009. Acoustic diode: rectification of acoustic energy flux in onedimensional systems. Phys. Rev. Lett. 103, 104301.

Madeo A., 2015. Generalised Continuum Mechanics and Engineering Applications. ISTE Press, London.

Maldovan M., 2013. Sound and heat revolution in phononics. Nature 503, 209-217.

Metrikine A.V., 2006. On causality of the gradient elasticity models. J. Sound. Vib. 297, 727-742.

Milton G.W., Briane M., Willis J.R., 2006. On cloaking for elasticity and physical equations with a transformation invariant form. New J. Phys. 8, 248.

Mindlin R.D., Tiersten H.F., 1962. Effects of couple-stresses in linear elasticity. Arch. Ration. Mech. Anal. 11, 415-488.

Nicorovici N.A., McPhedran R.C., Botten L.C., 1995. Photonic band gaps for arrays of perfectly conducting cylinders. Phys. Rev. E 52, 1135-1145.

Norris, A. N., Shuvalov A. L., 2011. Elastic cloaking theory. Wave Motion, 48, 525-538.

Pendry J.B., 2000. Negative refraction makes a perfect lens. Phys. Rev. Lett. 85, 3966-3969.

Porubov A.V., 2003. Amplification of Nonlinear Strain Waves in Solids. World Scientific, Singapore.

Samsonov A.M., 2001. Strain Solitons in Solids and How to Construct Them. CRC Press, Boca Raton.

Sánchez-Palencia E., 1980. Non-homogeneous media and vibration theory. Springer-Verlag, Berlin, 1980.

Sigalas M.M., Economou E.N., 1993. Band structure of elastic waves in two dimensional systems. Solid State Commun. 86, 141-143. 
Soubestre J., Boutin C., 2012. Non-local dynamic behaviour of linear fibre reinforced materials. Mech. Mater. 55, 16-32.

Toupin R.A., 1962. Perfectly elastic materials with couple stresses. Arch. Ration. Mech. An. 11, $385-414$.

Vasiliev A.A., Dmitriev S.V., Miroshnichenko A.E., 2010. Multi-field approach in mechanics of structural solids. Int. J. Solids Struct. 47, 510-525.

Voigt W., 1887. Theoretische Studien fiber die Elastizitatsverhiltnisse der Kristalle. Abh. Ges. Wissen. Göttingen 34, 3-100.

Wang P., Lu L., Bertoldi K., 2015. Topological phononic crystals with one-way elastic edge waves. Phys. Rev. Lett. 115, 104302.

Zheludev N.I., Kivshar Yu.S., 2012. From metamaterials to metadevices. Nature Materials 11, 917924. 